\title{
Poroviscoelasticity and compression-softening of agarose hydrogels
}

\author{
Abderrahim Ed-Daoui ${ }^{1}$, and Patrick Snabre ${ }^{2 *}$ \\ ${ }^{1}$ Laboratoire de Physique Théorique, de Particules, Modélisation et Energétique (LPTPME), Faculté des \\ sciences, Université Mohammed Premier, Oujda, Morocco. \\ ${ }^{2}$ Centre de Recherche Paul Pascal (CRPP), Université Bordeaux 1, CNRS (Centre National de la recherche \\ scientifique), UMR 5031, 115 Avenue Schweitzer, 33600 Pessac, France. \\ * Corresponding author \\ Email patrick.snabre@crpp.cnrs.fr
}

\begin{abstract}
Agarose hydrogels are poroviscoelastic materials that exhibit a waterlogged crosslinked microstructure. Despite an extensive use in biotechnologies and numerous studies of the elastic properties of agarose gels, little is known about the compressible behaviour and the microstructural changes of such fibrillar hydrogels under compression. The present work investigates the mechanical response of centimeter-sized pre-molded agarose cylinders when applying a compressive strain ramp over an extended range of loading speed and polymer concentration. One of the original contributions is the simultaneous monitoring of the changes in the hydrogel volume to determine the Poisson's ratio through a spatiotemporal method. The linear poroelastic response of agarose hydrogels shows a compressible behaviour at strain rates less than $0.7 \% \mathrm{~s}^{-1}$. The critical compressive strain of a few percent at the onset of the non linear regime and the always positive Poisson's ratio decrease when applying a slow compressive ramp. The mechanical response in the linear regime is typical of a deformation mode either dominated by the bending of semiflexible strands (enthalpic regime) or by the stretching of the network (entropic regime) at higher agarose concentration. Cyclic linear shear deformations superimposed to a compressive strain from $0.5 \%$ up to $40 \%$ further give evidence of a compression-softening of the network at the origin of the transition to the non linear regime whatever the network topology and connectivity. Finally, the buckling-induced aging of the network under a weak compression and the poroviscoelasticity of the hydrogel are shown to impact the relaxation of the normal stress and the equilibrium stress.
\end{abstract}

\section{Acknowledgment}

This work was supported by annual funding from the CNRS (Centre National de la Recherche Scientifique) in France and the University Mohammed Premier in Oujda (Morocco). The authors gratefully acknowledge M Benelmostafa for stimulating discussions, D Dahmani for supplying the Setexam agarose, the expertise center PLACAMAT in Talence (France) for the Cryo-SEM observations and B Mao (BioMérieux Craponne) for the molecular weight characterization of agarose samples by size exclusion chromatography.

Keywords Biopolymer hydrogel, Compression, Poroelasticity, Poisson's ratio, Buckling, Softening 


\section{Introduction}

Agarose is a linear neutral galactose polysaccharide containing a negligible amount of charged groups such as sulphate or pyruvate. Agarose often considered as a model biopolymer is extracted from the cell walls of red marine algae after an alkaline treatment of agarophytes and further removal of the more soluble agarose sulphate also referred as agaropectin (Djabourov et al. 2013; Zhang et al. 2019). Biopolymer hydrogels are suitable for a wide range of applications from food engineering as gelling agents (Mezzenga et al. 2005) to tissue engineering as porous scaffolds (Rinaudo 2008) and biotechnologies where uncharged agarose is widely used in gel electrophoresis (Kirkpatrick et al. 1993) and agar gel plates commonly serve as a growth medium for microorganisms (Divoux, Mao and Snabre 2015).

Agarose macromolecules exhibit a coil configuration in hot aqueous solutions with an overlap threshold polymer concentration of $0.5 \mathrm{wt} \%$ (San Biagio et al. 1984). As the temperature of the polymer solution is lowered below $55{ }^{\circ} \mathrm{C}$, a coil-helix transition occurs spontaneously and the agarose chains transform into relatively rigid fibrils (Arnott J et al. 1974; Hayashi et al. 1977, Foord and Atkins 1989; Schafer and stevens 1995) which aggregate by lateral association of 10-30 filaments to form polydisperse strands with diameters from $5 \mathrm{~nm}$ up to $50 \mathrm{~nm}$ and length from $0.15 \mu \mathrm{m}$ up to several micrometers depending on the agarose concentration (Laurent 1967; Dormoy and Candau 1991; Waki et al. 1982; Djabourov et al. 1989). The physical crosslinking of semiflexible strands through hydrogen bonds occurs below the gelation temperature of $40^{\circ} \mathrm{C}-35^{\circ} \mathrm{C}$ at a mass concentration as low as $0.1 \mathrm{wt} \%$ (Hayashi et al. 1977; Tokita and Hikichi 1987; Aymard et al. 2001) and gives rise to a fibrillar network that entraps a large amount of water. Many investigations have been carried out to clarify the growth kinetics of strands and the influence of the gelation conditions as the self-assembly of biopolymers involves an interplay between conformational changes, phase separation and molecular crosslinking (Feke and Prins 1974; Manno and Palma 1997; Xiong et al. 2005). A slow cooling rate or a low agarose concentration $c<1 \mathrm{wt} \%$ indeed promotes phase separation into polymer-rich and polymer-poor regions since water is not a good solvent for agarose (Morita et al. 2013). On the other hand, direct gelation without spinodal demixing is observed in the high concentration regime $c>2 \mathrm{wt} \%$ as phase separation becomes kinetically inhibited by crosslinking (San biagio et al. 1996). Recent normal force controlled rheology experiments supports the idea that lower cooling rates only delay the gelation process without affecting the terminal elastic properties of a $1.5 \mathrm{wt} \%$ agar hydrogel at ambient temperature (Mao et al. 2016)

Agarose hydrogels keep a constant volume without any significant swelling when placed in a relatively poor solvent as water and thus offer a suitable system for studying the mechanical properties of semi flexible biopolymer networks. Agarose hydrogels further display a sponge-like microstructure with a broad distribution of void spaces that becomes narrower as the polymer concentration increases (Righetti et al. 1981; Pernodet et al. 1997; Maaloum et al. 1998; Nitta et al. 2003). The mechanical properties of agarose and biopolymer hydrogels have been extensively studied and the concentration dependence of the Young modulus of the porous waterlogged material assumed as incompressible is reported to obey scaling laws with elastic exponents depending on the concentration regime (Watase and Nishinari 1983; Clark and Ross-Murphy 1987; Tokita and Hikichi 1987; Ramzi et al. 1998; Mohammed et al. 1998; Normand et al. 2000; Aymard et al. 2001; JolyDuhamel et al. 2002). However, little information is available concerning the influence of the loading speed on the Young modulus and the compressibility of the hydrogel.

Similar to a waterlogged sponge, an agarose hydrogel under a mechanical stress undergoes an elastic deformation and then water sweeps out from the compressible gel under the action of the applied stress. Kaneda and Iwasaki (2015) recently established a correlation between the time relaxation of the normal stress and water exudation from a $1.5 \mathrm{wt} \%$ agarose hydrogel at a low compressive strain of $5 \%$. The mechanical behaviour of hydrogels indeed strongly depends upon time and length scales involved in both the transport process of water and the reorganization of the network as predicted by the linear poroelastic theory.

The theory of linear poroelasticity was first introduced by von Terzaghi (1925) and extended later by Biot (1941) to describe the settlement of soils before being applied to the osmotic swelling and the mechanical compression of articular cartilage (Armstrong et al. 1984) and hydrogels (Armstrong et al. 1984; Yamaue and Doi 2005; Yamaue and Doi 2004; Doi 2009; Cai et al. 2010; Hu et al. 2010). The extent of water exudation from a constrained hydrogel and the time relaxation of the normal stress are closely related both to the elastic deformation and the compressibility of the soft material.

Hydrogels may further display some viscoelasticity and thus exhibit a time-dependent elastic behaviour as the network relaxes in response to an applied compressive strain (Hu and Suo 2012; Strange et al. 2013). Most of works on the poroviscoelasticity of biopolymer hydrogels assume a transiently incompressible material when appyling a fast compression strain ramp before holding a constant compressive strain without special attention to non linear effects (Strange et al 2013; Caccavo et al. 2017). Studies of agarose hydrogels generally misregard the influence of both the strain amplitude and the strain rate of the compressive ramp on the poroviscoelastic response. The few experimental contributions with different loading velocities address the stress-induced fracture of hydrogels (Nakamura et al. 2001; Normand et al. 2003; Forte et al. 2015). Interestingly, a 
compression-softening of fibrin networks has recently been reported due to the elastic buckling of the network under compression (Kim et al 2014). The weakness of a fibrillar network in the compressive direction can thus give rise to early non linear effects and have a significant impact on the mechanical properties of agarose hydrogels. Recent numerilcal simulations of the shear deformation of $3 \mathrm{~d}$ semiflexible networks indeed highlight a strain-softening mechanism dependent on the network topology but limited to enthalpic ansd sparsely connected strands (Bouzid et Del Gado 2018).

The present paper focuses on the coupling between the mechanical response of agarose hydrogels under compression, the squeezing out of water from the network and the compression-induced microstructural changes. Centimeter-sized pre-molded cylindrical agarose hydrogels were compressed between impermeable and frictionless rigid glass plates by applying a compressive strain ramp over a wide range of loading speeds from $0.1 \mu \mathrm{m} / \mathrm{s}$ up to $1 \mathrm{~mm} / \mathrm{s}$ and polymer concentrations from $0.3 \mathrm{wt} \%$ up to $23 \mathrm{wt} \%$. Changes in the volume of the hydrogel under compression were further monitored with a digital camera using a homemade spatiotemporal analysis (Mao et al. 2017a) to determine the Poisson's ratio in the linear regime. The accuracy of unconfined uniaxial compression experiments requires a precise adjustment of the parallelism of the compression plates as well as pre-molded hydrogels with perfectly controlled cylindrical geometry. From this point of view, microscale or macroscale indentation tests are easier to implement but unfortunately less suited to a study of the mechanical properties of hydrogels as the Young modulus cannot be measured without an a priori estimate of the Poisson's ratio in the linear regime (Hay and Wolf 2001). The non linear elastic behaviour of agarose gels under compression was finally investigated through the measurement of both the critical compressive strain at the onset of the non linear regime and the storage shear modulus of equilibrium hydrogels at high compressive strains up to $40 \%$.

The paper is organized as follows. Section 2 first describes the preparation of pre-molded agarose hydrogels and the Cryo-Scanning Electron Microscopy (Cryo-SEM) observations before introducing the unconfined uniaxial compression methods. The different protocols used to investigate the shear rheology of constrained hydrogels are then presented, namely the Small Amplitude Oscillatory Shear (SAOS) measurements and the shear stress relaxation tests in the linear shear regime. Section 2 ends with the presentation of the spatiotemporal method used to determine the Poisson's ratio of hydrogels in the linear regime. Sections 3.1 and 3.2 show the compressive stress - strain curves and discuss the influence of the loading speed and the agarose concentration on both the Young modulus and the Poisson's ratio of hydrogels using the linear poroelasticity theory. Section 3.3 provides an interpretation of the results in terms of the network microstructure through the enthalpic and entropic elasticity of fibrillar networks first introduced by Jones and Marques (1990) and Ramzi et al. (1998). Section 3.4 focuses on the physical mechanism at the origin of the transition to the nonlinear regime in relation with the compression-softening of agarose hydrogels. Finally, section 3.5 examines the viscoelasticity of agarose hydrogels on longer timescale and discusses the influence of both the buckling-induced aging of the network and the poroviscoelasticity of the hydrogel on the relaxation of the normal stress and the equilibrium stress on long timescale.

\section{Materials and methods}

2.1 Preparation of agarose hydrogels

Agarose powders with a sulphate content less than $0.2 \%$ were supplied by Setexam (type SHF, Lot S18063, Morocco) and Sigma-Aldrich (type EEO, Lot SLBW0321). A size exclusion chromatography method coupled with low-angle laser light scattering (Mitsuiki et al. 1999) was used to determine the molecular weight distribution of polysaccharides (average molecular weight $M_{w}=1.8810^{5} \mathrm{~g} / \mathrm{mol}$ and $M_{w}=3.0510^{5} \mathrm{~g} / \mathrm{mol}$ for Setexam and Sigma agarose, respectively). Agarose solutions were prepared by mixing the agarose powder with cold milli-Q water $\left(17 \mathrm{M} \Omega \mathrm{cm}\right.$ at $\left.25^{\circ} \mathrm{C}\right)$ and boiling at $100^{\circ} \mathrm{C}$ for $5 \mathrm{~min}$ with continuous magnetic stirring. Some water may be added in the bottle to compensate water evaporation. The clear hot solution was then kept at $(80 \pm 1)^{\circ} \mathrm{C}$ for one hour in a climate chamber (Binder MK53) for complete polymer dissolution and bubble removal. Solutions with a high agarose mass fraction $\alpha>9 \mathrm{~g}$ per $100 \mathrm{ml}$ become highly viscous and the formation of many bubbles under stirring made the conventional method inappropriate. In a way similar to the hydrothermal method proposed by Ayyad et al. (2010), the cold mixture was placed in a closed Pyrex bottle and heated at $110^{\circ} \mathrm{C}$ for 30 min under mild stirring in a climate chamber until the solution became clear. Heating at high temperature for too long time is not recommended due to thermal hydrolysis and intramolecular oxidation of agarose molecules with a consequent alteration in the mechanical properties of agarose gels (Mao et al. 2017b). An agarose mass fraction of $30 \mathrm{~g}$ per $100 \mathrm{ml}$ represents the upper limit for the preparation of homogeneous highly concentrated agarose hydrogels.

Mechanical characterization of hydrogels using standard compression tests requires special care to ensure a perfect parallelism of the top and bottom surfaces of the pre-molded bubble-free hydrogels. A Duralumin cylindrical mold (internal radius $R=7 \pm 0.01 \mathrm{~mm}$, height $H=14 \pm 0.01 \mathrm{~mm}$, surface roughness $4 \mu \mathrm{m} \pm 2 \mu \mathrm{m}$ as 
determined with a Contour Elite Bruker profilometer) was thus placed on a smooth glass surface (surface roughness $0.53 \mathrm{~nm} \pm 0.1 \mathrm{~nm}$ ) to prevent any adhesion of the hydrogel on the bottom surface and the mold was further sealed with an external bead of adhesive paste. The hot aqueous solution of agarose was then poured into the mold with a slight excess of liquid and immediately sealed with a circular glass coverslip before gelation occured under natural cooling at $20^{\circ} \mathrm{C}$ (Fig. 1a) after a time period of about 7 min as determined with a thermocouple immersed in the gel $\left(d T / d t \approx-5^{\circ} \mathrm{C} \mathrm{min}^{-1}\right)$. After 1 hour curation at $20^{\circ} \mathrm{C}$, the glass coverslip was gently slid off and the agarose cylinder was carefully removed from the mold (Fig. 1) before placing the hydrogel sample in a tank for 30 min equilibration and starting the compression test.
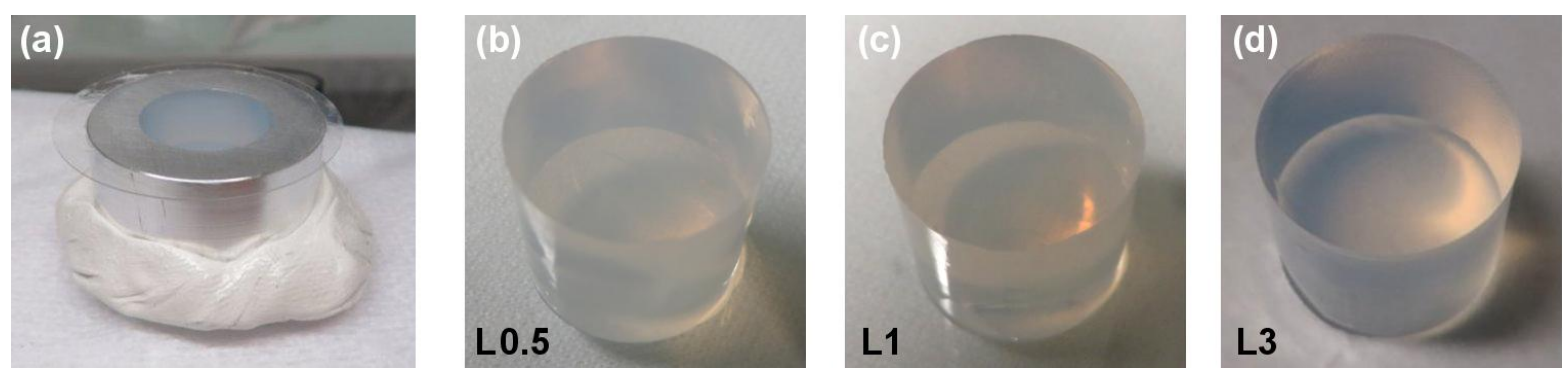

Fig. 1 (a) Duralumin cylindrical mold (internal radius $R=7 \pm 0.01 \mathrm{~mm}$, height $H=14 \pm 0.01 \mathrm{~mm}$ ) placed on a smooth glass plate; filled with a hot agarose solution $\left(\approx 80^{\circ} \mathrm{C}\right)$ and soon covered with a circular glass coverslip (a circular bead of adhesive paste seals the lower part of the mold). Pre-molded agarose cylinders L1.5 (b), L 3

(c) and L9 (d) after 1 hour curing at ambient temperature and subsequent removal of the hydrogels from the molds. Setexam agarose hydrogels with a polymer mass concentration $c \approx 1.48 \mathrm{wt} \%$ ( L1.5), $c \approx 2.91 \mathrm{wt} \%$ ( L 3 ) or $c \approx 8.26 \mathrm{wt} \%$ ( L9).

Aqueous solutions of agarose with a powder mass fraction $\alpha$ in the range from $0.5 \mathrm{~g}$ up to $30 \mathrm{~g}$ per $100 \mathrm{ml}$ water were prepared. Agarose hydrogels will be referred to as $\mathrm{L} \alpha$ or $\mathrm{H} \alpha$ with $\mathrm{L}$ or $\mathrm{H}$ standing either for Setexam samples (of lower $M_{w}$ ) or Sigma samples (of higher $M_{w}$ ) and $\alpha$ for the powder mass added in $100 \mathrm{ml}$ water (see Table 1 for the respective values of $\alpha$ en $g$ per $100 \mathrm{ml}$ and the concentration $c$ in $\mathrm{wt} \%$ ). The agarose powder density $\rho_{s} \approx(1.65 \pm 0.01) \mathrm{g} / \mathrm{cm}^{3}$ was measured by pycnometry. Agarose helices are stabilized by hydrogen bonds with water molecules occupying the inner part of the helix (Foord and Atkins 1989). As the mass fraction $\omega$ of agarose in hydrated agarose fibers is about $60 \%$ (Laurent 1967; Waki et al. 1982), the density of hydrated fibers is $\rho_{h}=\rho_{s} \omega+\rho_{o}(1-\omega) \approx 1.39 \mathrm{~g} / \mathrm{cm}^{3}\left(\rho_{O}=1 \mathrm{~g} / \mathrm{cm}^{3}\right.$ is the density of water), in close agreement with the value $\rho_{h} \approx 1.4 \mathrm{~g} / \mathrm{cm}^{3}$ reported by Arnott et al. (1974). Considering a value $\rho_{s} \omega \approx 1 \mathrm{~g} / \mathrm{cm}^{3}$ coincidently close to unity, the relationship $\phi=\alpha /\left(\alpha+100 \rho_{S} \omega / \rho_{o}\right) \approx \alpha /(\alpha+100)$ is therefore used to convert a mass fraction $\alpha$ of agarose in $100 \mathrm{ml}$ water into a volume fraction $\phi$ of hydrated fibers. As a consequence, the fiber volume fraction $\phi$ is close both to the agarose mass concentration $c=\alpha /(\alpha+100)$ in $\mathrm{wt} \%$ and to the helix volume fraction since optical rotation experiments suggest a very low non-helical content in agarose hydrogels at room temperature (Hayashi et al. 1977; Schafer and Stevens 1995; Djabourov et al. 2013)

\begin{tabular}{|c|c|c|}
\hline$\alpha(\mathrm{g} / 100 \mathrm{ml})$ & $c(\mathrm{wt} \%)$ & $\phi(\%)$ \\
\hline 0.3 & 0.299 & 0.299 \\
\hline 0.5 & 0.497 & 0.497 \\
\hline 0.7 & 0.695 & 0.695 \\
\hline 1 & 0.99 & 0.99 \\
\hline 1.5 & 1.48 & 1.48 \\
\hline 2 & 1.96 & 1.96 \\
\hline 3 & 2.91 & 2.91 \\
\hline 4 & 3.85 & 3.85 \\
\hline
\end{tabular}

\begin{tabular}{|c|c|c|}
\hline$\alpha(\mathrm{g} / 100 \mathrm{ml})$ & $c(\mathrm{wt} \%)$ & $\phi(\%)$ \\
\hline 5 & 4.76 & 4.76 \\
\hline 7 & 6.54 & 6.54 \\
\hline 9 & 8.26 & 8.26 \\
\hline 12 & 10.7 & 10.7 \\
\hline 15 & 13 & 13 \\
\hline 20 & 16.7 & 16.7 \\
\hline 25 & 20 & 20 \\
\hline 30 & 23.1 & 23.1 \\
\hline
\end{tabular}

Table 1 Respective values of the mass $\alpha$ of agarose powder in $\mathrm{g}$ per $100 \mathrm{ml}$ water, the polymer concentration $c$ in $\mathrm{wt} \%$ and the fiber volume fraction $\phi$ in \% for all the agarose samples L $\alpha$ and H $\alpha$ considered in the present work. 


\subsection{Cryo-Scanning Electron Microscopy}

The microstructure of agarose hydrogels was observed by cryogenic low vacuum scanning electron microscopy (JEOL 6700F). The chamber is cooled down to $T \approx-90^{\circ} \mathrm{C}$ and the sample cut in situ with a scalpel. The temperature is increased at a rate of $5^{\circ} \mathrm{C} / \mathrm{min}$ up to $\mathrm{T} \approx-50^{\circ} \mathrm{C}$ and kept constant for $5 \mathrm{~min}$ to sublime the water frozen inside the gel. The temperature is decreased back to $T \approx-85^{\circ} \mathrm{C}$ before coating the sample with a nanolayer of gold-palladium. The sample is finally cooled down to $T \approx-160^{\circ} \mathrm{C}$ and observed in the low vacuum chamber of the microscope using a secondary electron detector (SEI mode) at an acceleration voltage of $5 \mathrm{kV}$. The micrographs in Fig. 2 show fibrillar type networks with a broad pore size distribution and a typical mean pore diameter of $1 \mu \mathrm{m}$ at $c \approx 0.3 \mathrm{wt} \%$ and $0.3 \mu \mathrm{m}$ at $c \approx 3 \mathrm{wt} \%$ for Setexam samples (Fig. 16 in appendix $5.1)$.
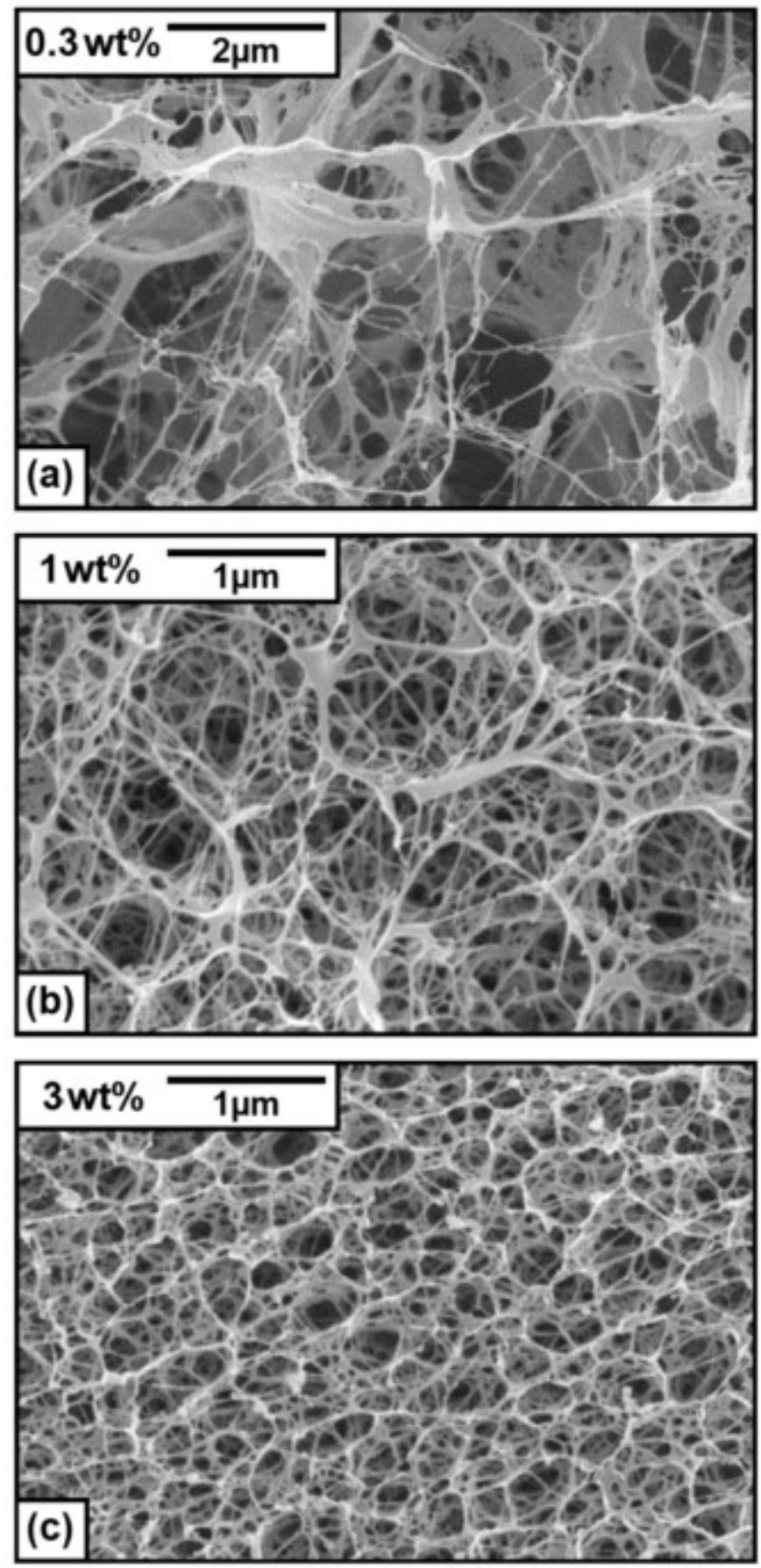

Fig. 2 Cryo-SEM micrographs of L0.3 ( $c \approx 0.3 \mathrm{wt} \%)(\mathbf{a}), \mathrm{L} 1(c \approx 0.99 \mathrm{wt} \%)(\mathbf{b})$ and L3 ( $c \approx 2.91 \mathrm{wt} \%)$ (c) Setexam agarose hydrogels. Samples are imaged using a secondary electron detector (SEI mode) at an acceleration voltage of $5 \mathrm{kV}$. 


\subsection{Unconfined compression}

Compression experiments of agarose hydrogels were performed at room temperature using either a CT3 4500G Brookfield texture analyzer ( $45 \mathrm{~N}$ loading cell, force precision $5 \mathrm{mN}$, speed accuracy $0.1 \%$ ) or a DHR2 TA Instruments rheometer $(50 \mathrm{~N}$ force transducer, force precision $1 \mathrm{mN}$, space resolution $0.1 \mu \mathrm{m})$. The premolded hydrogel placed in the central part of a rectangular tank is compressed either in air or immersed in water after 30 min equilibration (Fig. 3).
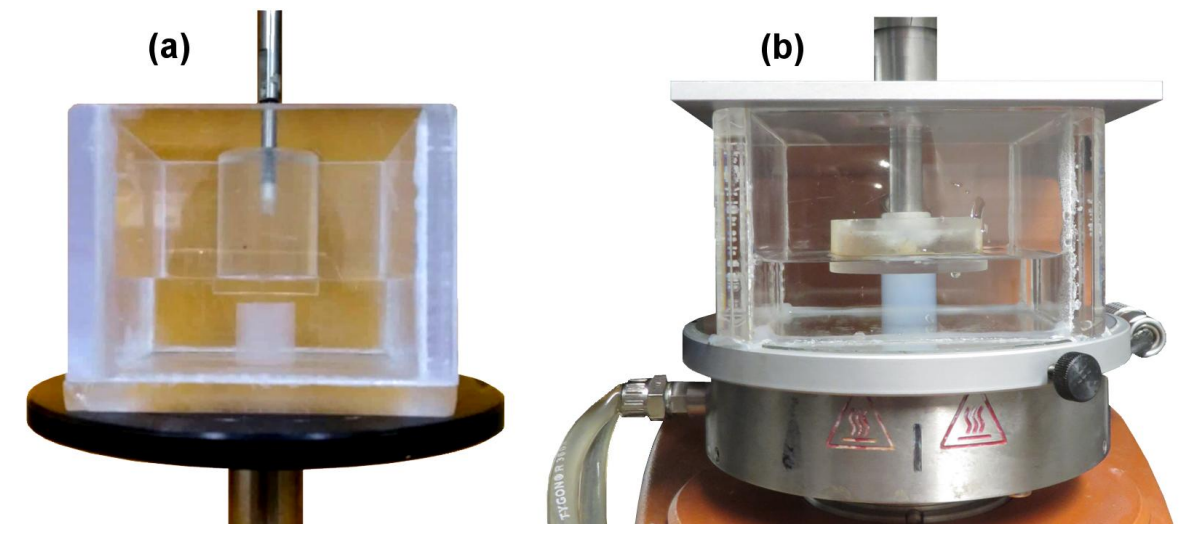

Fig. 3 Unconfined uniaxial compression of cylindrical agarose hydrogels immersed in water using either the Brookfield texture analyzer (a) or the DHR2 rheometer (b).

A rigid plate with a circular central hole covers the tank to prevent water evaporation from the hydrogel during equilibration and subsequent compression. An upper circular glass plate with an area larger than the hydrogel contact area was lowered at a constant speed $100 \mu \mathrm{m} / \mathrm{s}$ for the Brookfield texture analyzer ( 100 points $/ \mathrm{s}$, space accuracy $0.1 \mu \mathrm{m} / \mathrm{s}$ ) or over five decades of loading speed from $0.1 \mu \mathrm{m} / \mathrm{s}$ up to $10^{3} \mu \mathrm{m} / \mathrm{s}$ for the DHR2 rheometer ( 1 point/s up to 100 points/s, space accuracy $0.1 \mu \mathrm{m} / \mathrm{s}$ ) to cover a wide range of deformation timescales. The exact parallelism of the glass plates was previously adjusted by finely orienting the base plate. Top and bottom surfaces of the hydrogel in contact with the smooth glass plates were frictionless, so that the gel can uniformly expand during the compression test without any barrelling deformation (Fig. 3). A barrel-like deformation of a constrained hydrogel physically or chemically clamped to the plates would require a less precise averaging of the varying diameter over the height of the sample to determine the Poisson's ratio (Kaneda and Iwaski 2015; Kaneda 2018).

The moving plate was approached close to the upper surface of the equilibrium gel and the normal force was set to zero before starting the compressive strain ramp. A uniform downward motion of the upper plate was imposed and the compressive force $F(t)$ opposed by the gel cylinder of height $h(t)$ was monitored as a function of time. The initial height $h_{o}$ of the stress-free hydrogel was determined from the vertical position of the upper plate at which the applied load $F(t)$ suddenly rises ${ }^{1}$. The buoyancy force $F_{b}(t)=\pi C^{2}\left[h_{o}-h(t)\right] \rho_{o}$ exerted on the upper cylindrical glass plate of radius $C(C=12.5 \mathrm{~mm}$ or $C=20 \mathrm{~mm}$ for the compressive cell of either the texture analyzer or the DHR2 rheometer, respectively) was further subtracted from the applied load $F(t)$ when considering the unconfined compression of the hydrogel fully submerged in water. Here, the buoyancy force often overlooked in previous works somewhat influences the effective compressive force $F(t)-F_{b}(t)$ of soft hydrogels with an elastic modulus less than $300 \mathrm{kPa}$.

The compressive strain $\varepsilon$ is obtained by dividing the upper plate displacement $d h=h_{o}-h$ by the height $h_{o}$ of the unconstrained material and the stress $\sigma$ by dividing the effective load $F-F_{b}$ by the contact area $A_{o}(\varepsilon=0)$ of the equilibrium gel. The so-called engineering stress $\sigma=\left(F-F_{b}\right) / A_{o}$ is close to the true stress $\left(F-F_{b}\right) / A$ in the linear regime as the cross-sectional area $A$ of the compressed cylinder increases by less than $5 \%$ under a compressive strain $\varepsilon<5 \%$.

${ }^{1}$ The equilibrium height $h_{o}$ of the pre-molded gel is slightly less than the height $H$ of the duralumin mold as the sol-gel transition induces a small water release and a volume retraction of the sample as reported by Mao et al. (2016). Here, the hydrogel under tension in the mold at room temperature undergoes a uniform spontaneous shrinkage when demolding the cylinder with an equilibrium contraction ratio $\chi=\left(H-h_{o}\right) / H$ which increases linearly with the polymer concentration and reaches a plateau value $\chi=(3.1 \pm 0.2) \%$ at agarose mass concentration $c>1.5 \mathrm{wt} \%$. 
A $15 \%$ strain ramp was applied with a constant strain rate $d \varepsilon / d t$ from $7.110^{-4} \% \mathrm{~s}^{-1}$ up to $7.1 \% \mathrm{~s}^{-1}$ (compression speed in the range from $0.1 \mu \mathrm{m} / \mathrm{s}$ up to $10^{3} \mu \mathrm{m} / \mathrm{s}$ ) . No macroscopic fracture was observed below a critical strain rupture of about $30 \%$. The Young modulus was determined from the slope of the linear elastic region of the stress-strain curve. The intersection point of two asymptotic lines is usually considered to characterize the criçtical compressive strain $\varepsilon_{c}$ at the onset of the non linear regime but such a method significantly overestimates the strain threshold $\varepsilon_{c}$ and pronounced non linear features further make the choice the high strain asymptote difficult. For this reason, a $\pm 5 \%$ stress deviation of $(\sigma, \varepsilon)$ data from the linear fit was considered to determine the critical compressive strain $\varepsilon_{c}$ at the transition to the non linear regime with a relative accuracy $\pm 10 \%$.

\subsection{Shear rheology}

Shear stress relaxation and Small Amplitude Oscillatory Shear (SAOS) measurements were conducted at $20^{\circ} \mathrm{C}$ using the rotational DHR2 rheometer to investigate the impact of the compressive strain $\varepsilon$ upon the shear viscoelasticity of the agarose gel cylinder fully immersed in water. The shear experiments were performed after holding a static compressive strain for 15 min so that the hydrogel reaches a new quasi-equilibrium state and water exudation weakly influences the shearing of the constrained material. Prior to the shear tests, SAOS deformations superimposed to a low applied compressive strain $\varepsilon \approx 4 \%$ were probed at a frequency from $0.1 \mathrm{~Hz}$ up to $10 \mathrm{~Hz}$ to check the linearity of the response. The oscillatory stress resulting from a small amplitude harmonic shear strain $\gamma<510^{-2} \%$ is representative of linear viscoelastic behavior with a weak dependence upon the frequency and no significant slippage of the gel cylinder at the contact interface with the glass plates.

Shear stress relaxation experiments were performed on a weakly compressed quasi-equilibrium hydrogel ( $\varepsilon \approx 4 \%$ ) by superimposing a constant shear strain in the range from $510^{-2} \%$ down to $510^{-3} \%$ depending on the elastic modulus and the agarose mass concentration $0.5 \mathrm{wt} \% \leq c \leq 23 \mathrm{wt} \%$ (a shear strain $\gamma=510^{-3} \%$ corresponds to a radial displacement $\delta r(r=R) \approx \gamma H \approx 0.7 \mu \mathrm{m}$ of the outer upper surface of the gel cylinder and an angular deformation $\delta \theta=\delta r / R \approx 0.1 \mathrm{mrad}$ of the upper contact area of the gel cylinder to compare with the much less angular displacement resolution of the DHR2 rheometer of about $\left.10^{-5} \mathrm{mrad}\right)$. Strain hardening effects only become significant above a shear strain deformation of a few percent for agarose hydrogels (Bertula et al. 2019) and can be considered as negligible in the present experiments. A second order generalized Maxwell model consisting of a linear spring of elastic modulus $G_{1}$ connected in parallel with two Maxwell units (a spring of elastic modulus $G_{\mathrm{i}}$ connected in series with a dashpot of coefficient $\eta_{\mathrm{i}}$ with $\mathrm{i}=2,3$ ) was considered to describe the linear viscoelastic response and the time relaxation of the dimensionless shear stress modulus $G(t)=\tau(t) / \gamma$ of the assembly:

$G(t)=G_{1}+\sum_{\mathrm{i}=2}^{\mathrm{i}=3} G_{\mathrm{i}} \mathrm{e}^{-t / t_{\mathrm{i}}}=G_{o}+\sum_{\mathrm{i}=2}^{\mathrm{i}=3} G_{\mathrm{i}}\left(\mathrm{e}^{-t / t_{\mathrm{i}}}-1\right)$

where the instantaneous shear modulus $G_{o}=\sum_{\mathrm{i}=1}^{\mathrm{i}=3} G_{\mathrm{i}}$ of the hydrogel and the retarded shear moduli $\left(G_{2}, G_{3}\right)$ is determined using a non linear fit.

SAOS measurements were further carried out on an equilibrium hydrogel under a compressive strain $\varepsilon$ gradually increasing from $0.5 \%$ up to $40 \%$ by successive step $\mathrm{d} \varepsilon \approx 0.5 \%$ for $0.5 \%<\varepsilon<5 \%$ and $\mathrm{d} \varepsilon \approx 1.5 \%$ for $\varepsilon>5 \%$. After an elemental compressive strain $d \varepsilon$ of the hydrogel at a speed $d h / d t=100 \mu \mathrm{m} / \mathrm{s}$ $\left(d \varepsilon / d t=0.71 \% \mathrm{~s}^{-1}\right)$, the vertical strain $\varepsilon$ was hold for 1 hour and a cyclic shear strain of low amplitude $\gamma=210^{-2} \%$ was subsequently superimposed for 20 cycles at a frequency of $1 \mathrm{~Hz}$ to determine the storage modulus $G^{* *}(\varepsilon)$ and the loss modulus $G^{\prime *}(\varepsilon)$ of the equilibrium gel in the linear shear regime (the superscript asterix refers to an equilibrium state). Successive compressive strain ramps and hold periods were repeated to investigate the shear viscoelastic properties of equilibrium agarose hydrogels under compression.

Finally, the dynamics of water sweeping out from the hydrogel was investigated in the low compression regime. The time relaxation of the normal force $\sigma(t)$ was monitored after a fast compression of the hydrogel at $d h / d t=100 \mu \mathrm{m} / \mathrm{s}$ when holding a constant compressive strain $\varepsilon \approx 2 \%$ for $30 \mathrm{~min}$ to neglect at best any non linear effects. The dimensionless normal load relaxation $F(t)=\sigma(t) / \sigma_{o}$ was analyzed using an empirical stretched exponential function (Kaneda et al. 2015):

$F(t)=F^{*}+\left(1-F^{*}\right) \mathrm{e}^{-\left(t / t^{*}\right)^{S}}$ with $F^{*}(t)=\sigma^{*} / \sigma_{o}$ 
where $\sigma_{o}(t=0)$ is the normal stress immediately after the fast strain ramp, $\sigma^{*}$ the equilibrium normal stress at long timescale and $s$ the stretching exponent. A non linear fit of $\left[\sigma(t) / \sigma_{o}, t\right]$ data gives the reduced equilibrium stress $\sigma^{*} / \sigma_{o}$, the characteristic poroviscoelastic time $t^{*}$ and a stretching exponent $s \approx 0.6$.

\subsection{Poisson's coefficient}

The side view of the agarose hydrogel was monitored using a webcam (Logitech HD Pro c920). Image stacks were recorded at a frequency adjustable from 50 frames per second down to 1 image per minute depending on the duration of the compression test. The image acquisition started as soon as the upper plate moves down and image stacks were processed using a homemade plugin developed in java with the image processing software Image J (Schneider et al. 2012; Mao et al. 2017a) to determine the Poisson's ratio $v$ of the hydrogel within the limit of low compressive strains $0 \leq \varepsilon \leq 5 \%$.

Fig. 4 shows the images of L3 agarose gels in air or in water when applying a $15 \%$ strain ramp at a constant speed $d h / d t=100 \mu \mathrm{m} / \mathrm{s}\left(d \varepsilon / d t \approx 0.71 \% \mathrm{~s}^{-1}\right)$ or $d h / d t=0.1 \mu \mathrm{m} / \mathrm{s} \quad\left(d \varepsilon / d t \approx 7.110^{-4} \% \mathrm{~s}^{-1}\right)$. The constrained hydrogels undergo a lateral expansion (along the horizontal $x$ axis) regardless of the altitude $z$ with no sign of frictional barrelling for $0 \leq \varepsilon \leq 5 \%$ (Fig. 4). The Poisson's ratio $v=-\varepsilon_{x} / \varepsilon$ describes the relationship between the horizontal strain $\varepsilon_{x}$ perpendicular to the loading and the vertical strain $\varepsilon$ (Greaves et al. 2011).

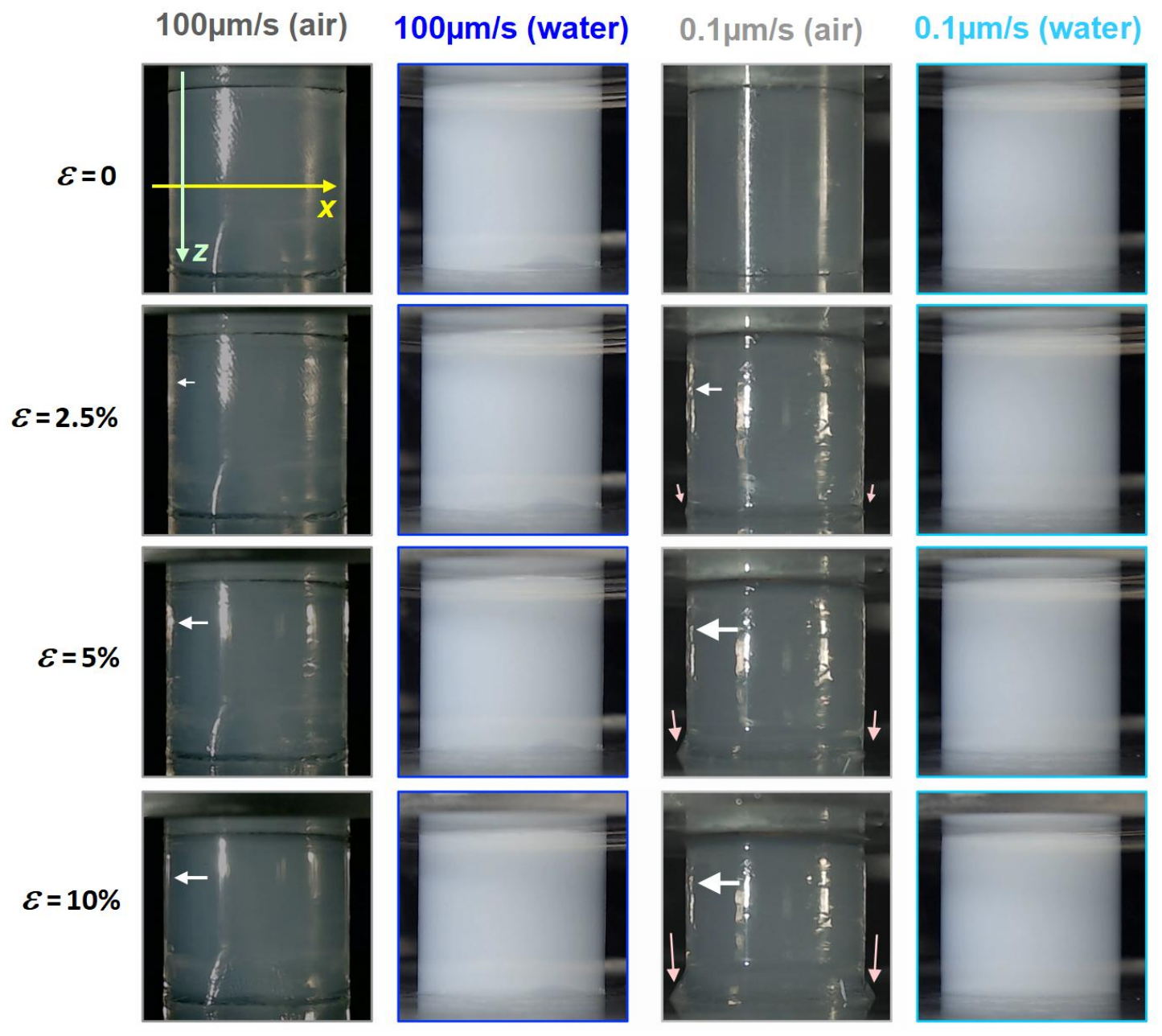

Fig. 4 Vertical compression of L3 hydrogels in air or in water when lowering the upper glass plate along the vertical direction $z$ with a loading speed $d h / d t=100 \mu \mathrm{m} / \mathrm{s}$ (two left columns) or $d h / d t=0.1 \mu \mathrm{m} / \mathrm{s}$ (two right columns). The images from top to bottom show the gel at different times during a $15 \%$ strain ramp for a compressive strain $\varepsilon=0,2.5 \%, 5 \%$ and $10 \%$, respectively. Horizontal white arrows highlight changes in grey levels with respect to the initial image before compression as a consequence of the appearance of water droplets at the outer surface of the gel in air while vertical pink arrows show the progressive formation of a water bead at the bottom of the gel slowly compressed in air. Setexam agarose hydrogels with a polymer mass concentration $c \approx 2.9 \mathrm{wt} \%$. 
A spatiotemporal projection of grey levels in a thin vertical or horizontal region of interest (ROI) was performed to extract the two diagrams $S T(\varepsilon, z)$ and $S T(\varepsilon, x)$ that highlight respectively the vertical contraction and the lateral expansion of the hydrogel under compression (Fig. 5). The lateral strain $\varepsilon_{x}$ is approximated by the change in the cylinder diameter divided by the unconstrained hydrogel diameter. Therefore, the Poisson's ratio $v$ can be expressed as:

$v=-\frac{\varepsilon_{x}}{\varepsilon} \approx \frac{\left(m-m_{o}\right) / m_{o}}{\left(n_{o}-n\right) / n_{o}}$

where $n(\varepsilon)$ and $m(\varepsilon)$ respectively denote the height in pixels and the diameter in pixels of the constrained hydrogel with $n_{O}=n(\varepsilon=0)$ and $m_{O}=m(\varepsilon=0)$. The space resolution of 60 pixels $/ \mathrm{mm}$ ensures a relative accuracy $d v / v \approx 1 / d n+1 / d m \approx 7 \%$ for the determination of the Poisson's ratio with $d n=n-n_{o} \approx 40$ pixels and $d m=m-m_{o} \approx 20$ pixels in the case of an incompressible material $(v=0.5)$ and a compressive strain $\varepsilon=5 \%$.
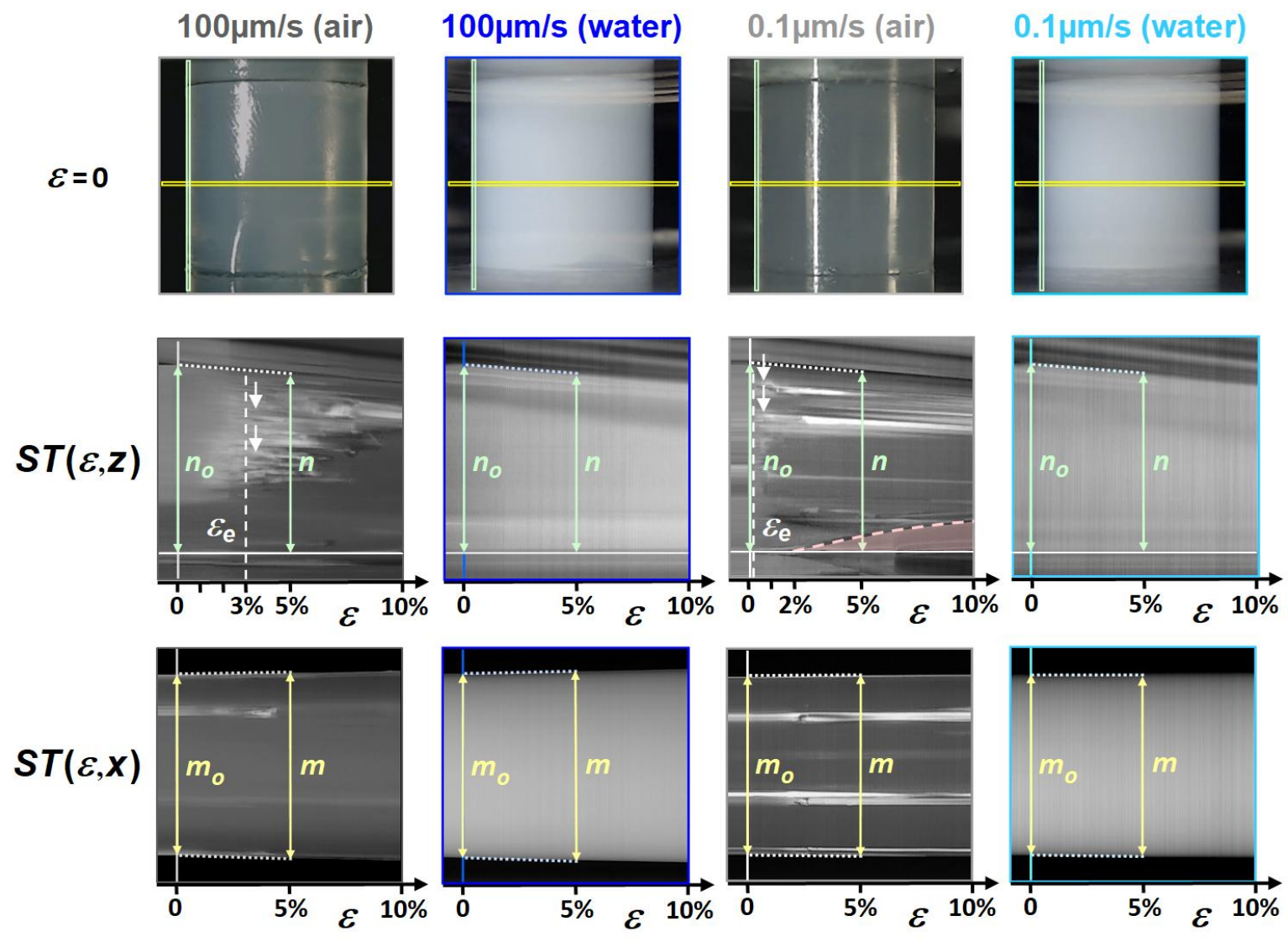

Fig. 5 Spatio temporal analysis of the stack images acquired when applying a $15 \%$ strain ramp with a loading speed $d h / d t=100 \mu \mathrm{m} / \mathrm{s}$ (two left columns) or $d h / d t=0.1 \mu \mathrm{m} / \mathrm{s}$ (two right columns) for L 3 hydrogels in air or in water. Spatio temporal diagrams $S T(\varepsilon, z)$ (second line) and $S T(\varepsilon, x)$ (third line) obtained from the vertical or the horizontal time projection of grey levels in the vertical green ROI or the horizontal yellow ROI drawn on top images. Dashed white lines in $S T(\varepsilon, z)$ diagrams highlight the formation of droplets at the air / gel interface above a critical strain $\varepsilon_{e}$ (the vertical white arrows in Fig.4 and Fig.5 highlight the changes in grey levels during water exudation) and the pink region delimited by a pink dotted line shows the growth of a water bead at the bottom of the gel cylinder slowly compressed in air (see pink vertical arrows in Fig.4). Dotted lines show either the vertical contraction in $S T(\varepsilon, z)$ diagrams or the radial expansion of the gel in $S T(\varepsilon, x)$ diagrams. The analysis of the spatio temporal diagrams from left to right gives Poisson's coefficients $v=0.478$ $(d h / d t=100 \mu \mathrm{m} / \mathrm{s}$ in air), $v=0.474(d h / d t=100 \mu \mathrm{m} / \mathrm{s}$ in water $), v=0.27(d h / d t=0.1 \mu \mathrm{m} / \mathrm{s}$ in air $)$ and $v=0.21(\mathrm{dh} / \mathrm{dt}=0.1 \mu \mathrm{m} / \mathrm{s}$ in water $)$. Setexam agarose hydrogels with a polymer mass concentration $c \approx 2.9 \mathrm{wt} \%$. 
The spatiotemporal analysis was further used to determine the drained Poisson's ratio $v *$ of the equilibrium constrained gel when holding a static compressive strain for one hour. Temporal changes in the cylinder diameter are first caused by the pressure build-up and the radial expansion of the hydrogel during a fast compression ramp and then by the water release in a characteristic time of a few minutes when holding a static compressive strain as evidenced by the spatiotemporal diagram $S T(\varepsilon, x)$ of the L3 sample in Fig. 6. The drained Poisson's ratio $v^{*}$ representative of the change in the water volume fraction is determined from the relation $v^{*} \approx\left(m^{*}-m_{o}\right) /\left(m_{o} \varepsilon\right)$ where $m^{*}$ is the diameter in pixels of the equilibrium drained hydrogel at long timescale.

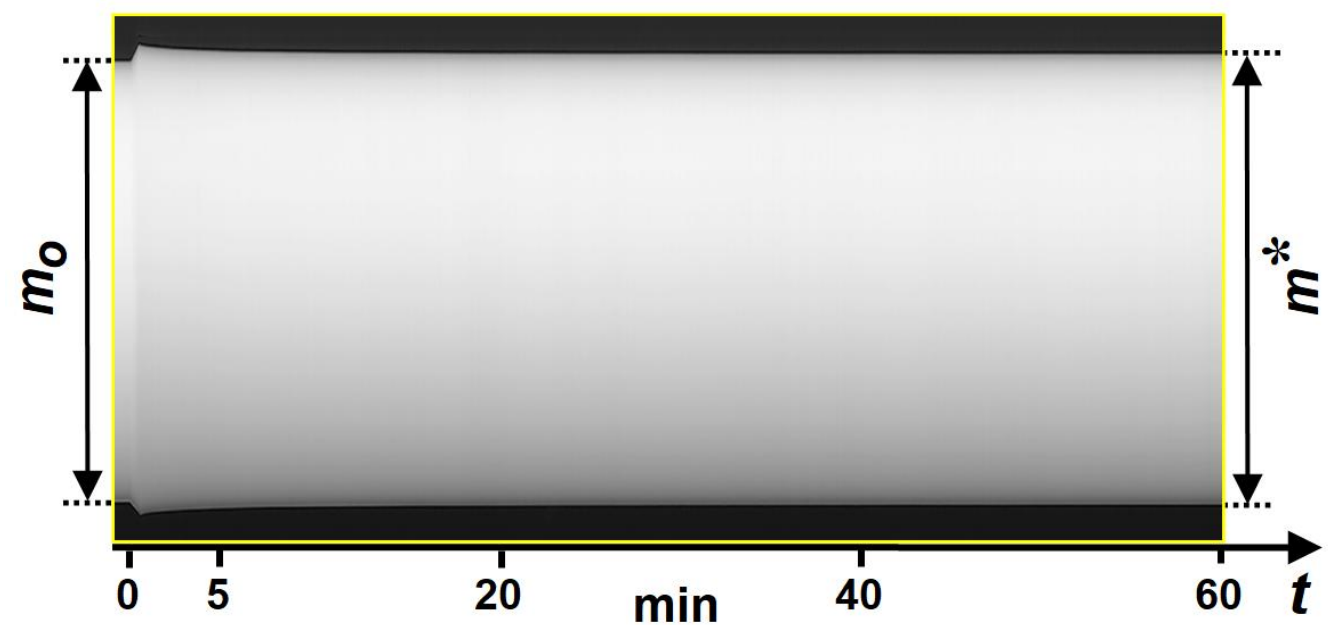

Fig. 6 Spatiotemporal diagram $S T(t, x)$ of an L 3 hydrogel in response to a $15 \%$ strain ramp at a loading speed $d h / d t=100 \mu \mathrm{m} / \mathrm{s} \quad(0 \leq t \leq 21 \mathrm{~s})$ followed by the relaxation of the sample at a constant compressive strain $\varepsilon=15 \%$ for 1 hour. The drained Poisson's ratio $v^{*}=\left(m^{*}-m_{o}\right) /\left(m_{o} \varepsilon\right) \approx 0.15 \pm 0.035$ is determined from the mreasurement of the diameter $m_{o}$ in pixels of the unconstrained gel and the diameter $m^{*}$ in pixels of the equilibrium drained hydrogel. Setexam agarose hydrogel with a polymer mass concentration $c \approx 2.9 \mathrm{wt} \%$.

\section{Results and discussion}

3.1 Compression and poroelasticity of agarose hydrogels

The present paragraph concerns both the fast and the slow compression bevaviour of L 3 hydrogels $(c \approx 2.9 \mathrm{wt} \%)$ in air or in water (Fig. 7). The stress - strain curves $\sigma(\varepsilon)$ display a first linear elastic region followed by a non linear response above a critical compressive strain $\varepsilon_{c}$ with either an upward or a downward concavity respectively when applying a $15 \%$ strain ramp at a loading speed $d h / d t=100 \mu \mathrm{m} / \mathrm{s}$ $\left(d \varepsilon / d t \approx 0.71 \% \mathrm{~s}^{-1}\right)$ or $d h / d t=0.1 \mu \mathrm{m} / \mathrm{s}\left(d \varepsilon / d t \approx 7.110^{-4} \% \mathrm{~s}^{-1}\right)($ Fig. 7$)$.

When deforming the L3 hydrogel at a fast loading speed $d h / d t=100 \mu \mathrm{m} / \mathrm{s}$ (Fig. 7a), the material behaves as a nearly incompressible material in the linear regime with a Poisson's ratio $\approx 0.47-0.48$ as determined from the spatiotemporal analysis of the cylinder deformation (first and second columns in Fig. 5). The spatiotemporal diagram $S T(\varepsilon, z)$ of the L3 sample rapidly compressed in air shows the formation of droplets at the air / gel interface at a strain $\varepsilon_{\mathrm{e}} \approx 3 \%$ (Fig. 5) close to the critical strain $\varepsilon_{\mathrm{c}} \approx 3.3 \%$ at the transition to the non linear regime (Fig. 7a).

In the opposite case of a slow loading speed $d h / d t=0.1 \mu \mathrm{m} / \mathrm{s}$ (Fig. 7b), the gel appears as softer with a lower Young modulus as the solvent is immediately squeezed out from the porous material $\left(\varepsilon_{e} \approx 0\right.$, white arrows in the $S T(\varepsilon, z)$ diagram from the third column in Fig. 5). The compressible hydrogel thus displays a lower Poisson's ratio of 0.21 in water and 0.27 in air $(S T(\varepsilon, x)$ diagrams from third and fourth columns in Fig. $5)^{2}$ and the non linear regime occurs above a critical strain $\varepsilon_{\mathrm{c}} \approx 0.5 \%-0.6 \%$ (Fig. $7 \mathrm{~b}$ ).

\footnotetext{
${ }^{2}$ Note that the slow compression of the L3 hydrogel in air causes an early exudation of water from the outer free surface (third column in Fig. 5) and an apparent slight increase in the rate of swelling of the cylinder with a consequent overestimation of the Poisson's ratio as determined by the spatiotemporal method.
} 

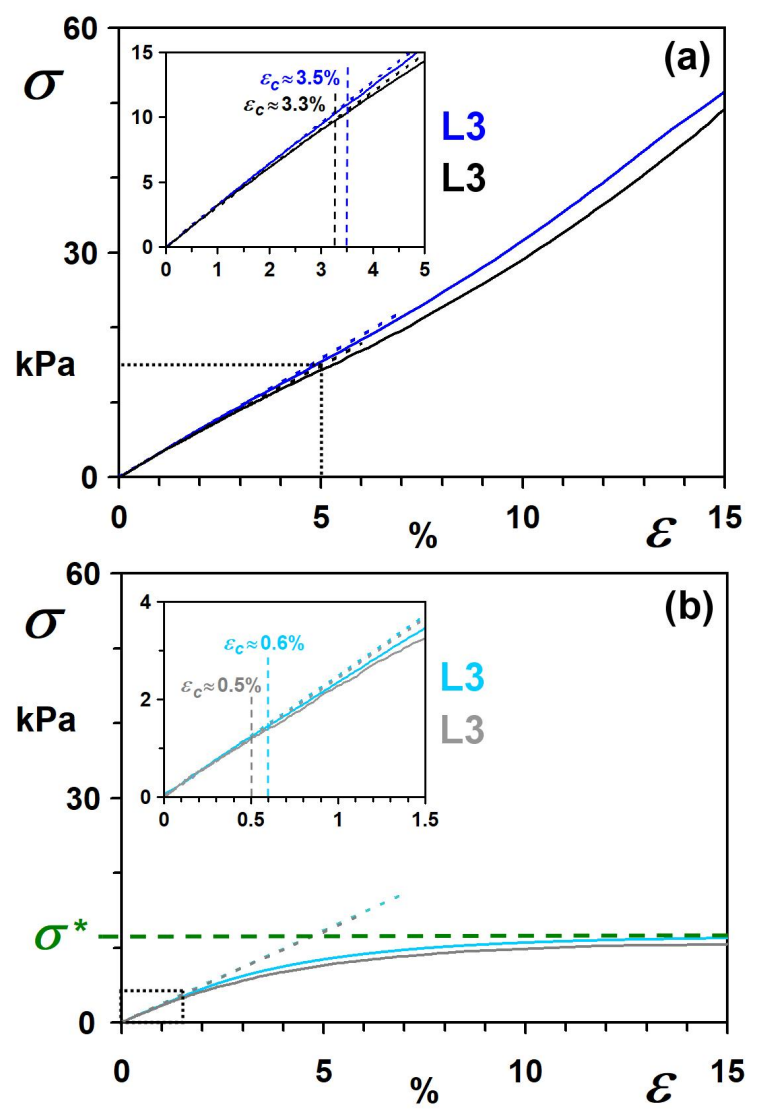

Fig. 7 Normal stress $\sigma$ versus the compressive strain $\varepsilon$ when applying a $15 \%$ strain ramp with a loading speed $d h / d t=100 \mu \mathrm{m} / \mathrm{s}$ (a) or $d h / d t=0.1 \mu \mathrm{m} / \mathrm{s}$ (b) for an L3 hydrogel in air (full black and grey curves) or in water (full dark blue and light blue curves). Dotted lines are best linear fits of $\sigma(\varepsilon)$ data at low compressive strain $\varepsilon<\varepsilon_{c}$ with the critical strain $\varepsilon_{c}$ shown in the inserts. The dashed green line in (b) shows the plateau stress $\sigma^{*}$ at high compressive strains. Setexam agarose hydrogels with a polymer mass concentration $c \approx 2.9 \mathrm{wt} \%$.

Interestingly, the typical two stage stress response of the hydrogel submitted to a slow compression ramp (Fig 7b) is reminiscent of the mechanical behaviour of an open-cell elastomeric foam compressed under quasistatic conditions with an elastic response at low compressive strain and a plateau stress $\sigma^{*}$ in the non linear regime (Ashby et al. 2005; Ouellet et al. 2006). Here, the plateau stress $\sigma^{*}$ is not representative of a plastic damage since the deformation of agarose hydrogels is nearly reversible (Fig. 17 in appendix 5.2) ${ }^{3}$. Furthermore, whether the gel is compressed in air or in water has no significant influence upon the stress - strain curves (Fig. 7) which is expected for an initially equilibrium hydrogel and further highlights the weakness of the interfacial tension forces in the water release process.

Such a behavior is consistent with the theory of linear poroelasticity (Yamaue and Doi 2005; Doi 2009; Cai et al. 2010). In response to a compressive strain ramp, the pressurization of the interstitial fluid induces a radial tensile stress that causes a lateral expansion of the hydrogel. A fast compression delays the release of water from the hydrogel that behaves as an incompressible material $(v=0.5)$ at short timescale and supports a compressive stress $\sigma=E_{o} \varepsilon$ in the linear regime where $E_{o}$ is the Young modulus of the undrained material. If the duration of the strain ramp in the linear regime exceeds the characteristic time $t^{*}$ of fluid permeation through the network, water exudation may occur very early and the compressible material $(v<0.5)$ appears as softer with a lower Young modulus $E=2 G_{o}(1+v)$ where the shear elastic modulus $G_{o}=E_{o} / 3$ of the hydrogel is mainly representative of the network microstructure (Yamaue and Doi 2005).

${ }^{3}$ The deformation of the agarose hydrogel appears as reversible after a fast or a slow $15 \%$ compressive strain ramp and the material nearly recovers the initial shape within half an hour when removing the load. The hydrogel can further be compressed up to a $90 \%$ strain without breaking under an extremely low loading speed $d h / d t=0.1 \mu \mathrm{m} / \mathrm{s} \quad\left(d \varepsilon / d t \approx 7.110^{-4} \% \mathrm{~s}^{-1}\right)$ at agarose concentrations from $0.5 \mathrm{wt} \%$ up to $12 \mathrm{wt} \%$ as previously reported for gellan gels for polymer concentrations $0.81 \mathrm{wt} \%<c<2.5 \mathrm{wt} \%$ (Nakamura et al. 2001) . 
Both poroelastic and viscoelastic effects associated with the transport of the liquid and the reorganization of the network can influence the normal stress relaxation and the deformation process of the hydrogel. The shear stress relaxation of L3 hydrogels weakly compressed in water $(\varepsilon=4 \%)$ shows an instantaneous shear elastic modulus $G_{o} \approx 100 \mathrm{kPa}$ and a retarded viscoelasticity at short and long timescales in the range from a few tens of seconds up to half an hour (Fig. 8). The loading speed and the time duration of the compressive strain ramp weakly influences the viscoelastic response of the hydrogel (Fig. 8) as non linear effects remain negligible at a low compressive strain $\varepsilon \approx 4 \%$. As predicted by the linear poroelasticity theory, the elastic modulus $E /[2(1+v)]$ calculated from the measured values of both the Young modulus $E$ and the Poisson's ratio $v$ of L3 samples submerged in water is close to the shear modulus $G_{O}$ of the hydrogel independently of the loading speed (Table 2).

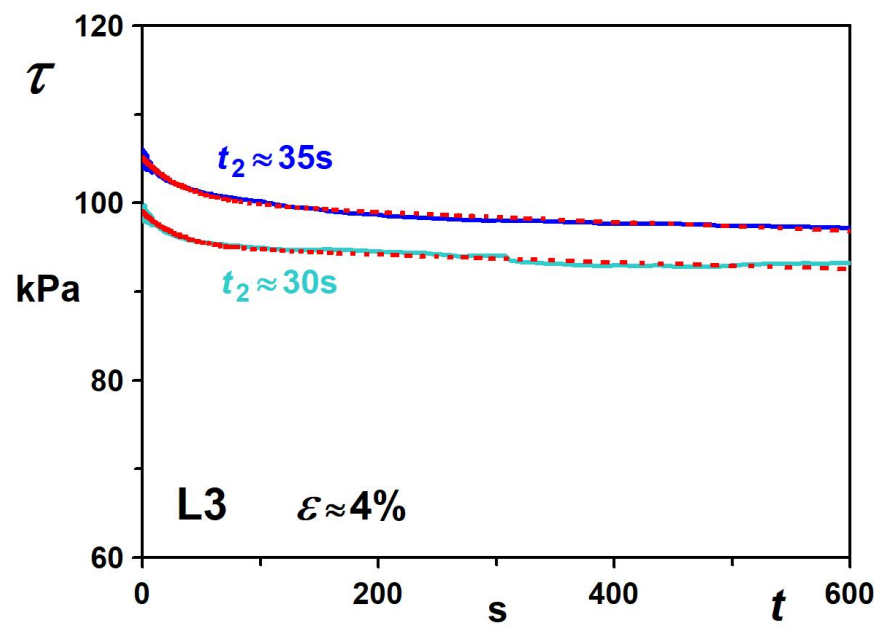

Fig. 8 Shear stress relaxation $\tau(t)$ of quasi-equilibrium L3 hydrogels weakly compressed in water when superimposing a static shear strain $\gamma=510^{-3} \%$. Before shearing the hydrogel, a $4 \%$ compressive strain ramp is imposed either rapidly ( $d h / d t=100 \mu \mathrm{m} / \mathrm{s}$, full dark blue curve) or slowly ( $d h / d t=0.1 \mu \mathrm{m} / \mathrm{s}$, full light blue curve) and the static strain $\varepsilon=4 \%$ is hold for $15 \mathrm{~min}$. Dotted red lines correspond to a second order generalized Maxwell model (Eq. 1) fitted to data with the parameter values: $G_{o}=105 \mathrm{kPa}, G_{2}=4.9 \mathrm{kPa}, G_{3}=11.7 \mathrm{kPa}$, $t_{2}=35 \mathrm{~s}, t_{3}=30 \mathrm{~min}$ for $d h / d t=100 \mu \mathrm{m} / \mathrm{s}$ and $G_{o}=99 \mathrm{kPa}, G_{2}=3.9 \mathrm{kPa}, G_{3}=9 \mathrm{kPa}, t_{2}=30 \mathrm{~s}$, $t_{3}=30 \mathrm{~min}$ for $d h / d t=0.1 \mu \mathrm{m} / \mathrm{s}$. Setexam agarose hydrogels with a polymer mass concentration $c \approx 2.9 \mathrm{wt} \%$.

\begin{tabular}{|c|c|c|c|c|c|}
\hline $\begin{array}{c}d h / d t \\
\mu \mathrm{m} / \mathrm{s}\end{array}$ & $\begin{array}{c}\varepsilon_{c} \\
(\%)\end{array}$ & $v$ & $\begin{array}{c}E \\
(\mathrm{kPa})\end{array}$ & $\begin{array}{c}G_{o} \\
(\mathrm{kPa})\end{array}$ & $\frac{E}{2 G_{o}(1+v)}$ \\
\hline 100 & 3.5 & 0.474 & 317.5 & 105 & 1.025 \\
\hline 0.1 & 0.6 & 0.21 & 247.1 & 99 & 1.033 \\
\hline
\end{tabular}

Table 2 Critical compressive strain $\varepsilon_{c}$, Poisson's ratio $v$, Young modulus $E$, elastic shear modulus $G_{o}$ and dimensionless ratio $E /\left[2 G_{o}(1+v)\right]$ of L3 samples in water when applying either a fast or a slow $15 \%$ strain ramp at a loading speed $d h / d t=100 \mu \mathrm{m} / \mathrm{s}$ or $d h / d t=0.1 \mu \mathrm{m} / \mathrm{s}$. Setexam agarose mass concentration $c \approx 2.9 \mathrm{wt} \%$.

3.2 Influence of compression speed and agarose concentration on Poisson's ratio

Compression experiments of L 3 hydrogels immersed in water were performed over 5 decades of loading speeds from $d h / d t=10^{3} \mu \mathrm{m} / \mathrm{s}\left(d \varepsilon / d t \approx 7.1 \% \mathrm{~s}^{-1}\right)$ down to $d h / d t=0.1 \mu \mathrm{m} / \mathrm{s} \quad\left(d \varepsilon / d t \approx 7.110^{-4} \% \mathrm{~s}^{-1}\right)(\mathrm{Fig}$. 9a). The Young modulus $E$, the Poisson's ratio $v$ and the critical compressive strain $\varepsilon_{c}$ display lower values at a loading speed less than $100 \mu \mathrm{m} / \mathrm{s}\left(d \varepsilon / d t \approx 0.71 \% \mathrm{~s}^{-1}\right)$ as water has time to sweep out from the hydrogel (Fig. $9 b)$. As reported in the previous paragraph, the elastic modulus $E /[2(1+v)]$ derived from compression experiments performed in triplicate take a value $E /[2(1+v)]=(106 \pm 4) \mathrm{kPa}$ close to that of the shear elastic modulus $G_{o}=(99 \pm 4) \mathrm{kPa}$ regardless of the imposed strain rate (Fig. 9b). The slightly lower value of the shear elastic modulus $G_{o}$ arises from weak nonlinear effects as a $4 \%$ compressive strain is hold for 15 min before running the stress relaxation test, as will be discussed later in section 3.4 . 

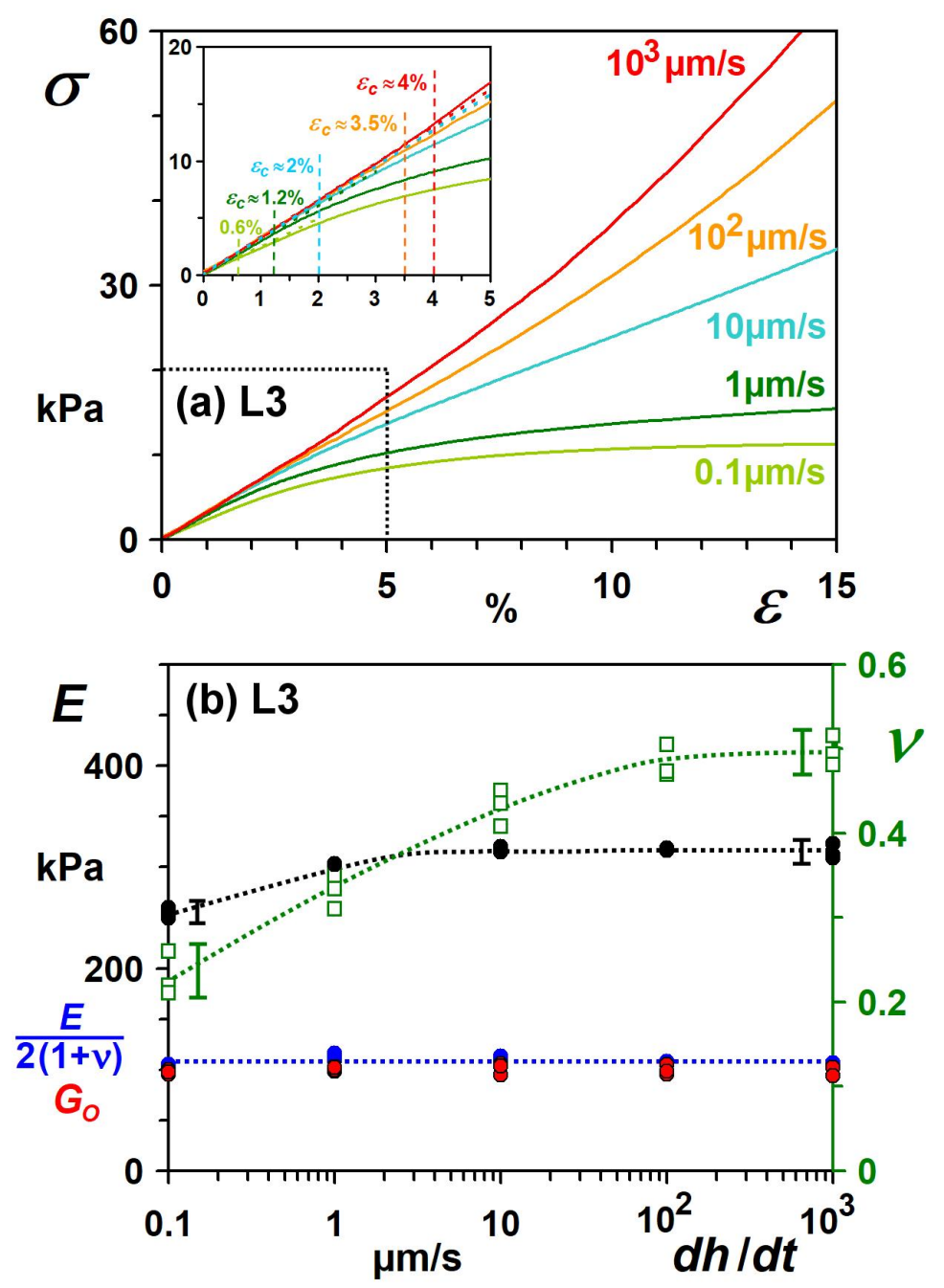

Fig. 9 (a) Normal stress $\sigma$ versus the compressive strain $\varepsilon$ for L3 hydrogels in water and different loading speed $d h / d t$ from $0.1 \mu \mathrm{m} / \mathrm{s}$ up to $10^{3} \mu \mathrm{m} / \mathrm{s}$ in the DHR2 rheometer. The insert is a zoom in the low strain region where dotted lines are best linear fits of $\sigma(\varepsilon)$ data in the limit of low strains $\varepsilon<\varepsilon_{c}$ and dashed vertical lines show the critical compressive strain $\varepsilon_{\text {c }}$. (b) Semi logarithmic plot of the Young modulus $E$ (full black circles) and the Poisson's ratio $v$ (open green squares) of L 3 hydrogels compressed in water versus the loading speed $d h / d t$. Full blue circles and full red circles respectively stand for the elastic modulus $E /[2(1+v)]$ calculated from $(E, v)$ data and the shear modulus $G_{o}$ determined from shear stress relaxation experiments after a $4 \%$ compressive strain ramp at a loading speed $d h / d t$ and a hold period of $15 \mathrm{~min}$. Compression and shear stress relaxation tests were performed in triplicate. Setexam agarose hydrogels with a polymer mass concentration $c \approx 2.9 \mathrm{wt} \%$.

Considering now a constant loading speed $d h / d t=100 \mu \mathrm{m} / \mathrm{s}$, the hydrogel becomes stiffer when increasing the agarose concentration with a corresponding significant decrease in the critical strain $\varepsilon_{c}\left(\varepsilon_{\mathrm{c}} \approx 4.5 \%\right.$ for L1 sample and $\varepsilon_{\mathrm{c}} \approx 1.55 \%$ for L30 sample, Fig. 10) which could be the consequence of a more intense pressurization of the internal liquid phase and an earlier water release from dense networks under fast compression. Surprisingly, the Poisson's ratio measured at the same compression speed $d h / d t=100 \mu \mathrm{m} / \mathrm{s}$ over a compressive strain range $0<\varepsilon<5 \%$ keeps fairly constant values $0.47<v<0.52$ representative of a nearly incompressible material without significant dependence on the agarose concentration (Fig. 11). Such an observation goes against an interpretation based on a water release to explain the decrease in the critical compressive strain $\varepsilon_{c}$ at high agarose concentration. Predicting the variations of the critical strain $\varepsilon_{c}$ is indeed far from trivial since the transition to the non linear regime may involve not only the competition between the pressure build-up and the permeation dynamics of water through the porous hydrogel but also a possible reorganization of the network under compression as will be discussed later in section 3.4. 

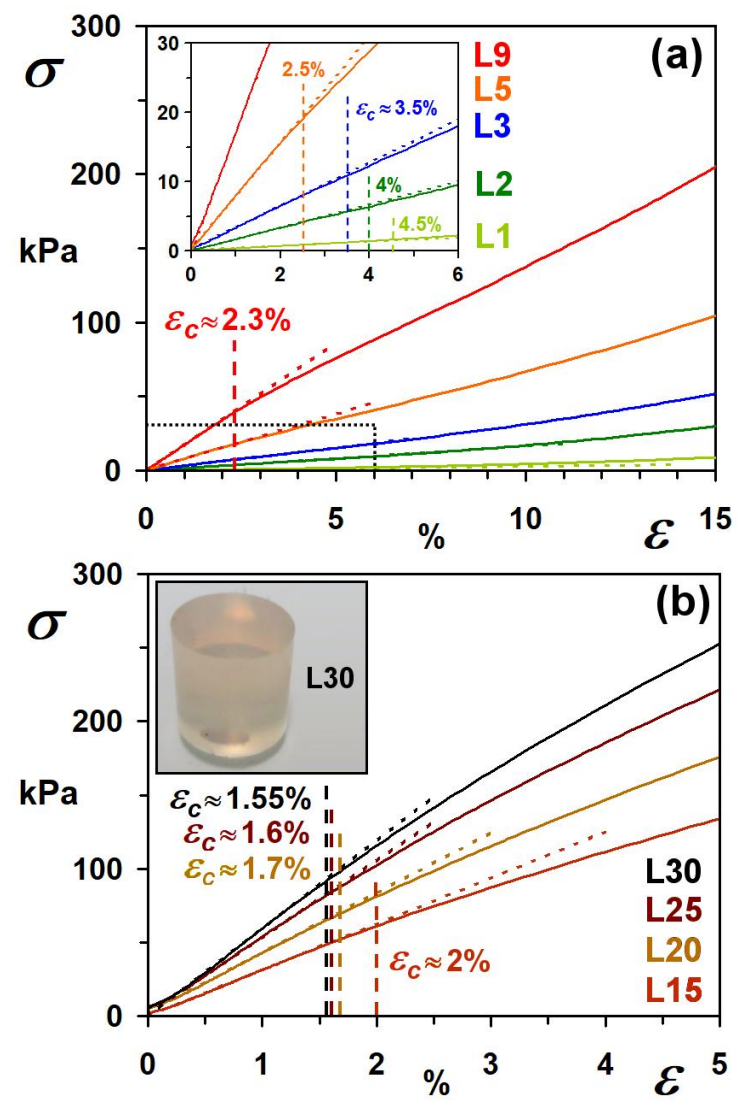

Fig. 10 Normal stress $\sigma$ versus the compressive strain $\varepsilon$ for $\mathrm{L} \alpha$ hydrogels with a mass fraction $1 \mathrm{~g} \leq \alpha \leq 9 \mathrm{~g}$ (a) and $15 \mathrm{~g} \leq \alpha \leq 30 \mathrm{~g}$ (b) of Setexam agarose powder per $100 \mathrm{ml}$ water when applying a $15 \%$ strain ramp at a loading speed $d h / d t=100 \mu \mathrm{m} / \mathrm{s}$ in the DHR2 rheometer. Dotted lines are best linear fits of $\sigma(\varepsilon)$ data in the limit of low strains $\varepsilon<\varepsilon_{c}$ and dashed vertical lines show the critical compressive strain $\varepsilon_{c}$. The insert in (a) is a zoom of the low strain region while the insert in (b) shows a picture of the agarose hydrogel L 30 with a polymer mass concentration $c \approx 23 \mathrm{wt} \%$.

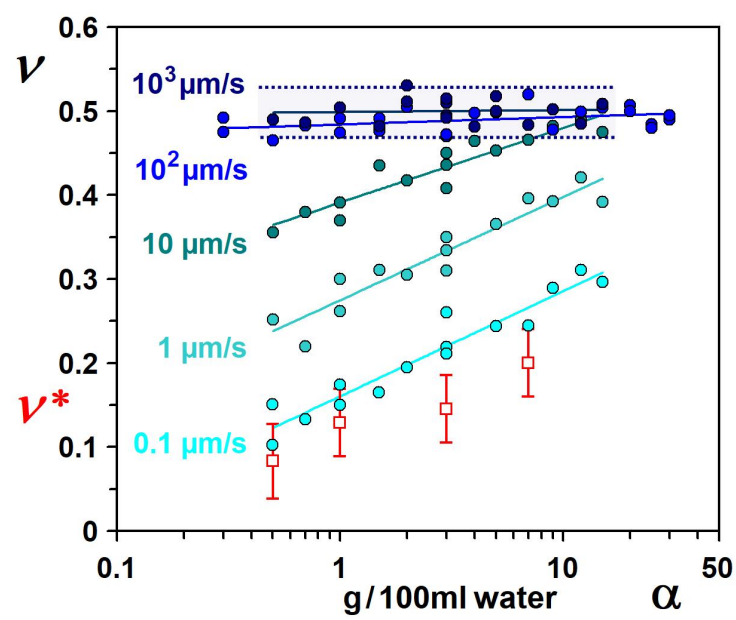

Fig. 11 Semi logarithmic plot of the Poisson's ratio $v$ of $L \alpha$ hydrogels versus the mass fraction $\alpha$ of Setexam agarose powder per $100 \mathrm{ml}$ water for several loading speed $d h / d t$ varying from $0.1 \mu \mathrm{m} / \mathrm{s}$ (full light blue circles) up to $10^{3} \mu \mathrm{m} / \mathrm{s}$ (full dark blue circles). The upper dotted dark blue rectangle highlights the dispersion of the Poisson's ratio values $v=0.499 \pm 0.033$ (16 data points) in the linear regime at high loading speed $d h / d t=10^{3} \mu \mathrm{m} / \mathrm{s}$ and the full color lines are the best logarithmic fits of $(v, \alpha)$ data for each of the loading speed. Open red square symbols stand for the drained Poisson's ratio $v^{*}$ of Setexam agarose equilibrium hydrogels determined from the spatiotemporal diagram $S T(\varepsilon, x)$ when holding a $4 \%$ static compressive strain for 1 hour. 
On the other hand, the hydrogel behaves as a compressible material with a Poisson's ratio $0.1<v<0.5$ when applying a slow strain ramp $(d h / d t<100 \mu \mathrm{m} / \mathrm{s})$ since water is swept out very early from the network on longer timescales (Fig. 11). The drained Poisson's ratio $v^{*}$ of the equilibrium constrained hydrogel determined from the spatiotemporal diagram $S T(\varepsilon, x)$ when holding a $4 \%$ static compressive strain for 1 hour increases with the agarose concentration from 0.1 up 0.2 (open red square symbols in Fig. 11). As expected, the agarose hydrogel under a very slow strain ramp at a loading speed $d h / d t=0.1 \mu \mathrm{m} / \mathrm{s}$ remains in a near equilibrium state as the Poisson's ratio $v$ in the linear regime is barely greater than the drained Poisson's ratio $v^{*}$ (Fig. 11). Note also that the positive drained Poisson's ratio $v^{*}$ of equilibrium hydrogels in the linear regime excludes any significant collapse or folding of semi-entrant cells as in the honeycomb or foam effect (Greaves et al 2011).

Agarose hydrogels yet behave very differently depending on the loading speed and whether the strain rate $d \varepsilon / d t$ is lower or higher than $0.71 \% \mathrm{~s}^{-1}$ ( $d h / d t=100 \mu \mathrm{m} / \mathrm{s}$ for centimeter-sized samples). Indeed, a strain rate $d \varepsilon / d t$ in the range from $0.5 \% \mathrm{~s}^{-1}$ up to $10 \% \mathrm{~s}^{-1}$ is usually considered in the literature to assume an incompressible behaviour of agarose hydrogels and determine the Young modulus $E_{O}$ in the linear regime (Ramzi et al. 1998; Normand et al. 2000; Aymard et al. 2001; Kaneda et al. 2015; Delaine-Smith et al. 2016). A minimum strain rate of $0.5 \% \mathrm{~s}^{-1}$ corresponds to a deformation period of a few seconds before water flows out from a centimeter-sized hydrogel and then induces a compressible behavior in the linear regime. The permeation dynamics of water over longer timescales is controlled by the imposed strain rate and the permeability of the network through the poroviscoelastic time $t^{*}$ of the hydrogel (see section 3.5).

\subsection{Elastic behaviour in the linear regime}

The present section concerns the linear elasticity of hydrogels in relation with the agarose concentration and the deformation mode of the microstructure. All the measurements of the elastic modulus $3 E /[2(1+v)]$ and the shear modulus $G_{o}$ carried out at different loading speed and agarose concentration were plotted in logarithmic scale as a function of the fiber volume fraction (Fig.12). For this purpose, the deviation $\left(\phi-\phi_{g}\right)$ from the transition threshold is introduced to establish the scaling laws governing the elastic behaviour of the hydrogel (Djabourov 1991) where $\phi_{g}$ is the fiber volume fraction at the gelation point. The gelation threshold of agarose hydrogels at $20^{\circ} \mathrm{C}$ has been reported above a typical critical mass concentration $c_{g}=0.07 \mathrm{wt} \%-0.13 \mathrm{wt} \%$ (Hayashi et al. 1977; Tokita and Hikichi 1987; Normand et al. 2000) much smaller than that of agarose coils in hot solutions because of the semiflexible structure of helical filaments and strands. Recently, a very nice investigation of the surface tension of water-agarose systems with a molecular weight $210^{5}-310^{5} \mathrm{~g} / \mathrm{mol}$ at concentrations from $10^{-3} \mathrm{wt} \%$ up to $0.2 \mathrm{wt} \%$ indicates a second order phase transition and the formation of a macroscopic gel phase above the critical concentration $c_{g}=0.1 \mathrm{wt} \%$ (Ichinose and Ura 2020). Therefore, a critical fiber volume fraction $\phi_{\mathrm{g}}=0.1 \%$ at the gelation threshold is considered to analyze the elastic behaviour of agarose hydrogels. Note that the introduction of the variable $\left(\phi-\phi_{g}\right)$ with $\phi_{g}=0.1 \%$ mainly influences the value of the scaling exponent in the percolation regime at low fiber volume fraction $\phi<10 \phi_{\mathrm{g}} \approx 1 \%$.

The concentration dependence of both the linear elastic modulus $E /[2(1+v)]$ and the shear modulus $G_{o}$ of Setexam agarose hydrogels gives evidence of two distinct power law regimes with an elastic exponent $\beta \approx 2.1 \pm 0.05$ for $0.4 \%<\phi<2.8 \%$ and $\beta \approx 1.35 \pm 0.05$ for $3.75 \%<\phi<23 \%$ (Fig. 12). The use of either Setexam or Sigma agarose powder of different average molecular weight has an impact on the elastic moduli scaling as $M_{w}^{0.8}$ but little influence upon the elastic exponents $\beta$ in both regimes as shown in Fig. 18 (appendix 5.3). Previous studies on the elasticity of agarose hydrogels with $10^{5} \mathrm{~g} / \mathrm{mol}<M_{w}<210^{5} \mathrm{~g} / \mathrm{mol}$ concerned a less extended range of concentrations $0.3 \mathrm{wt} \%<c<5 \mathrm{wt} \%$ but reported similar scaling exponents $\beta \approx 1.7-2.2$ below $2 \mathrm{wt} \%$ and $\beta \approx 1.5 \pm 0.1$ above $2 \mathrm{wt} \%$ (Rochas et al. 1994; Ramzi et al. 1998; Normand et al. 2000). The linear storage modulus of various gelatin hydrogels formed by the aggregation of semiflexible helical structures above a gelation threshold $\phi_{g} \approx 0.24 \%$ also obeys a two regime master curve with an elastic exponent $\beta \approx 2$ for $\phi<2 \%$ and $\beta \approx 1.5$ at higher helix volume fraction (Joly-Duhamel et al. 2002; Djabourov et al. 2013). Ramzi et al. (1998) and Joly-Duhamel et al. (2002) first introduced two types of linear elasticity to interpret the values of the scaling exponents: an enthalpic elasticity dominated by the bending of the semiflexible structural units in weak networks and an entropic elastic regime dominated by the stretching of flexible junctions between rods in stronger networks. 


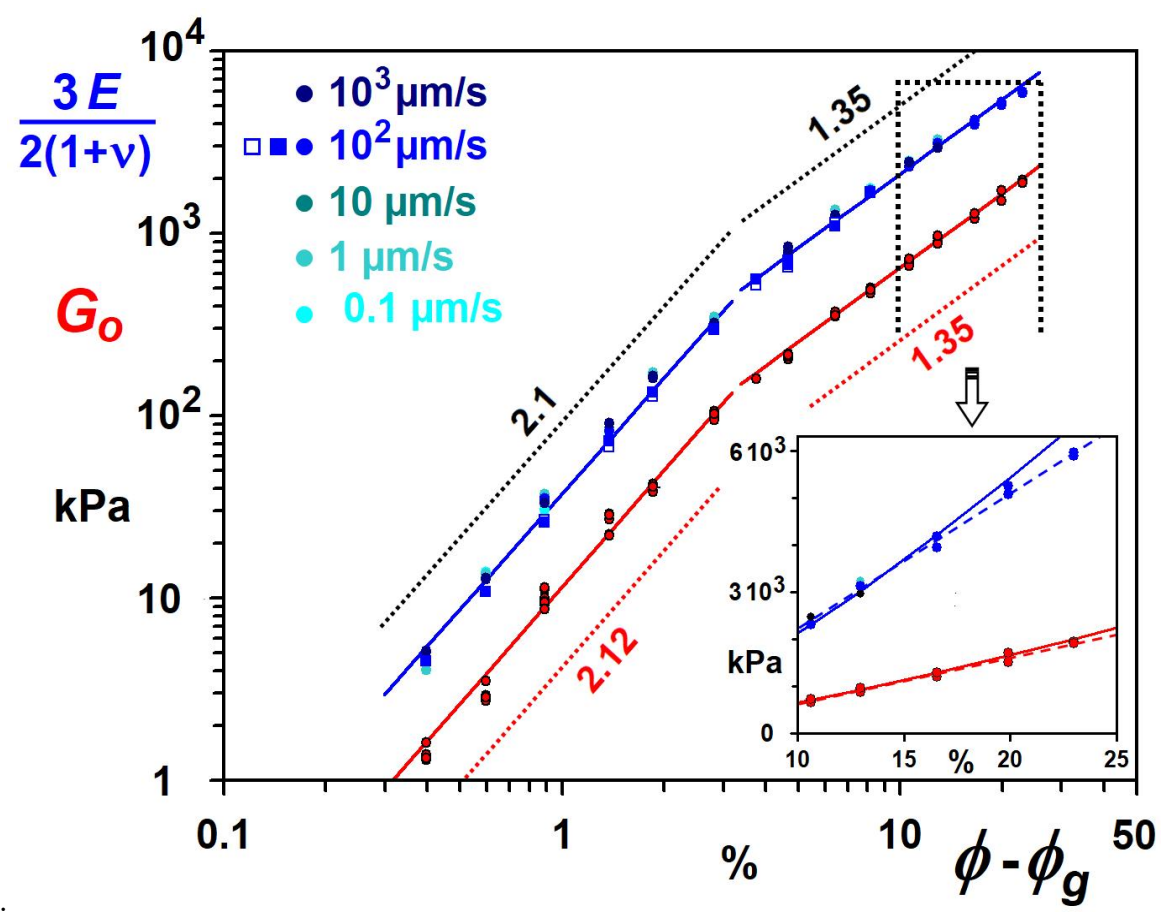

Fig. 12 Logarithmic plot of the elastic modulus $3 E /[2(1+v)]$ (full blue circles and blue squares) and the shear elastic modulus $G_{o}$ (full red circles) versus the fiber volume fraction $\phi-\phi_{g}$ in Setexam agarose hydrogels with $\phi_{g}=0.1 \%$. Full blue circles stand for compression experiments in the DHR2 rheometer performed in water at different loading speed from $0.1 \mu \mathrm{m} / \mathrm{s}$ (full light blue circles) up to $10^{3} \mu \mathrm{m} / \mathrm{s}$ (full dark blue circles). Full blue squares are for experiments conducted in the Brookfield texture analyzer either in air (open blue squares) or in water (full squares squares) at a loading speed $\mathrm{dh} / \mathrm{dt}=100 \mu \mathrm{m} / \mathrm{s}$. Full red circles stand for the elastic shear modulus $G_{o}$ obtained from shear stress relaxation tests in the DHR2 rheometer after a $4 \%$ fast compressive strain ramp. Full blue line and full red line are the best power law fits of $\left[3 E /(2+2 v), \phi-\phi_{g}\right]$ and $\left(G_{o}, \phi-\phi_{g}\right)$ data in the enthalpic $\left(0.4 \% \leq \phi-\phi_{\mathrm{g}} \leq 2.8 \%\right)$ and the entropic elastic regime $\left(3.75 \% \leq \phi-\phi_{\mathrm{g}} \leq 23 \%\right)$, respectively. Dotted blue and red lines highlight the respective slopes of power law fits with an indication of the value of the elastic exponent $\beta$. The insert is a zoom in linear coordinates of the high agarose concentration regime delimited by vertical dotted lines in the main figure. The dashed blue line and the dashed red line in the insert are the best linear fits of $\left[3 E /(2+2 v), \phi-\phi_{g}\right]$ and $\left(G_{o}, \phi-\phi_{g}\right)$ data for $10 \%<\phi-\phi_{\mathrm{g}}<23 \%$.

The aggregation of 10 - 20 agarose helices into semiflexible strands (Waki et al. 1982; Dormoy et Candau 1991) noticeably increases the persistence length $l_{p}$ (the typical length under which the chain is rod-like) of the structural units in the network. According to Molginer and Rubinstein (2005), a weakly cross-linked strand of $10-20$ helical filaments deviates from a linear rod over a critical length of $200 \mathrm{~nm}-300 \mathrm{~nm}$. The persistence length of multiple-helical polysaccharides as xanthan or $\kappa$-carrageenan aggregates typically lies in the range $200 \mathrm{~nm}-300 \mathrm{~nm}$ (Coviello et al. 1986; Meunier et al. 2000). Recent Scanning Electron Microscopy (SEM) and Atomic Force Microscopy (AFM) observations of agarose hydrogels at low polymer concentrations $c<1 \mathrm{wt} \%$ indeed revealed long-shaped strands of diameter $6 \mathrm{~nm}-20 \mathrm{~nm}$ with a persistence length of a few hundred nanometers and a coordination number (average number of strands connecting in a junction) $z \approx 3$ below the $3 \mathrm{~d}$ critical isostatic point $z_{c} \approx 6$ (Bertula et al. 2019). The linear storage modulus of fibrillar collagen hydrogels with a low coordination number $z \approx 3-3.5$ scales quadratically with the fiber volume fraction consistent with an enthalpic elasticity arising from non affine bending deformations of the network (Jansen et al. 2018). Furthermore, the phase separation (spinodal demixtion) that occurs during the cooling of agarose hydrogels at low agarose concentrations $c<2 \mathrm{wt} \%$ (San Biagio et al. 1996; Morita et al. 2013) promotes the formation of a sparsely connected and inhomogeneous network with an enthalpic elastic behaviour. A low coordination number and a persistence length smaller than the distance between junctions points results in a dominant bending deformation mode of the floppy network whereas affine stretching deformations arise above the critical isostatic threshold $z_{c}$ (Broedersz et al. 2011; Huisman et Lubensky 2011). 
In the bending dominated regime, the theory first proposed by Jones and Marques (1990) to describe the mechanical behaviour of cellular materials with an open cell network predicts an elastic exponent $\beta=\left(3+d_{f}\right) /\left(3-d_{f}\right)$ where $d_{f}$ is the fractal dimension of the structural units. The scaling exponent $\beta \approx 2.1 \pm 0.05$ derived from the concentration dependence of the linear elastic moduli at low agarose fiber volume fraction $0.5 \%<\phi<3 \%$ (Fig.12) yields a reasonable fractal dimension $d_{f} \approx 1.07 \pm 0.03$ representative of straight semiflexible strands ${ }^{4}$.

In the second regime, the average distance between the junction points becomes less than the persistence length of strands and the dense and homogeneous network exhibits an entropic elasticity. In the now commonly accepted context of junction zones composed of short flexible polysaccharide coils binding rigid rods at high helical concentrations (Dajabourov at al. 2013), the model proposed by Jones and Marques (1990) for the linear elasticity of the so called freely hinged network predicts an elastic exponent $\beta=3 /\left(3-d_{f}\right)$. A fractal dimension $d_{f} \approx 1$ of the rigid strands yields a scaling exponent $\beta=1.5$ not so far from the experimental value $\beta \approx 1.35 \pm 0.05$ at high fiber volume fraction $3 \%<\phi<23 \%$ (Fig. 12). Within the framework of an entropic elasticity, one may assume a linear elastic modulus proportional to the volume number of junctions, namely to the reciprocal $1 / \xi^{3}$ of the cube of the mean distance $\xi$ between crosslinks. In the limit of rigid strands with a radius $r$ much larger than the mesh size of the network, the typical spacing $\xi$ between crosslinks scales as $\left(\phi-\phi_{g}\right)^{-1 / 2}$ (Ogston 1958, Lang et al. 2013, and Fig.16 in appendix 5.1) ${ }^{5}$ and the elastic modulus $E \propto 1 / \xi^{3} \propto\left(\phi-\phi_{\mathrm{g}}\right)^{3 / 2}$ exhibits a scaling exponent $\beta=1.5$ identical to that derived from the model of Jones and Marques (1990). In the next paragraph, the concentration dependence of the critical compressive strain $\varepsilon_{c}(\phi)$ at high fiber volume fraction will provide an alternative explanation for the lower value $\beta \approx 1.35$ of the scaling exponent in the entropic elastic regime.

A critical look at Fig. 12 finally shows the existence of a third regime in the limit of very high agarose concentrations $c>10 \mathrm{wt} \%$. The insert in Fig.12 (zoom in linear coordinates) indeed gives evidence of elastic moduli $E /(1+v)$ and $G_{o}$ nearly proportional to the fiber volume fraction over the concentration range $10 \% \leq \phi-\phi_{\mathrm{g}} \leq 23 \%$. In highly concentrated agarose hydrogels, all the strands contribute to the elastic properties of the network and the linear elastic exponent then takes a unit value $\beta=1$. Such a rubber-like behaviour was not mentioned earlier because of the difficulty of preparing homogeneous highly concentrated agarose hydrogels.

\subsection{Microstructural changes in the non linear regime}

The non linear regime occurs during the strain ramp at a relatively small compressive critical strain $\varepsilon_{c}$ from a a few percent down to a few tenths of a percent depending both on the loading speed $d h / d t$ and the agarose fiber volume fraction (Fig. 13).

4 For highly diluted agarose and biopolymer hydrogels close to the percolation threshold ( $\phi_{\mathrm{g}} \approx 0.1 \%<\phi<0.5 \%$ ), experimental values of the elastic exponent $\beta$ are quite dispersed in the range from 1.8 up to 4 (Tokita and Hikichi 1987; Clark and Ross-Murphy 1987; Kawabata et al. 1996; Mohammed et al. 1998; Fujii et al. 2000; Gunasekaran and Yoon 2014). Considering agarose fibers as stiff linear rods, the scalar percolation theory from de Gennes (1980) gives a critical exponent $\beta \approx 1.9$ (Djabourov 1991). On the other hand, a vectorial percolation model taking into account the bending of fibers predicts a higher value $\beta \approx 3.96$ of the elastic exponent (Sahimi 1986). However, the dispersion in the experimental values of the scaling exponent in the close vicinity of the gel point also arises both from loose chains (free chains) that gradually vanish upon increasing the agarose concentration and from a greater sensitivity of the hydrogel elasticity to the molecular weight distribution of the polymer.

${ }^{5}$ One strand of length $\xi$ and cross section $r^{2}$ in a volume of size $\xi$ corresponds to a fiber volume fraction $\phi-\phi_{g} \propto r^{2} \xi / \xi^{3}=r^{2} / \xi^{2}$ and a mesh size $\xi \propto r\left(\phi-\phi_{g}\right)^{\lambda}$ with a scaling exponent $\lambda=-1 / 2$. The scaling relation $\xi \propto\left(\phi-\phi_{g}\right)^{-1 / 2}$ describes the concentration dependence of the pore diameter in agarose hydrogels both for the larger and the smaller free spaces at fiber volume fraction $\phi<3 \mathrm{wt} \%$ (Fig. 16 in appendix 5.1). 


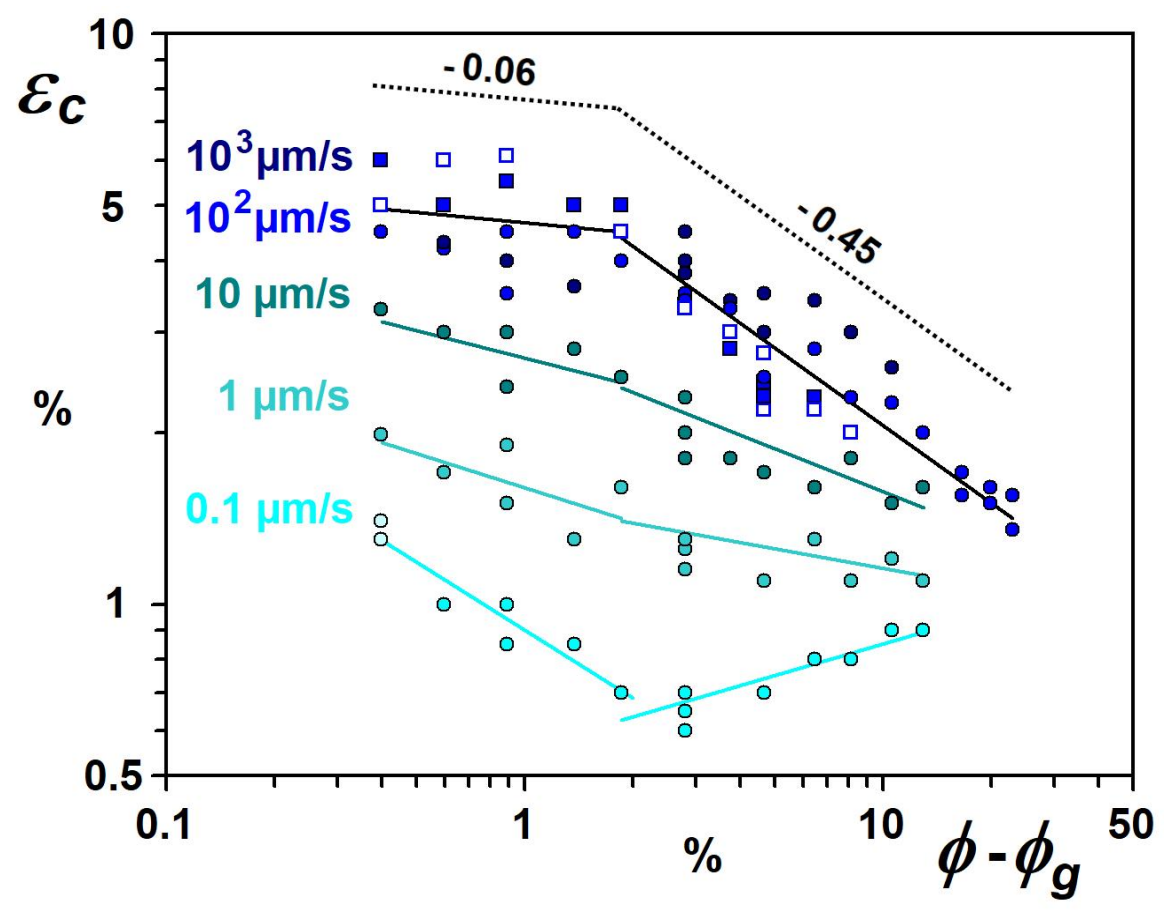

Fig. 13 Logarithmic plot of the critical strain $\varepsilon_{c}$ versus the fiber volume fraction $\phi-\phi_{g}$ in Setexam agarose hydrogels with $\phi_{\mathrm{g}}=0.1 \%$. Full blue circles and full blue squares of different colors refer to the same compression experiments and loading speeds as in Fig. 12. Full black lines are the best power law fits of $\left(\varepsilon_{c}, \phi-\phi_{g}\right)$ data for a fast compression of the hydrogel at a loading speed $d h / d t \geq 100 \mu \mathrm{m} / \mathrm{s}$ either for $0.4 \% \leq \phi-\phi_{\mathrm{g}} \leq 1.9 \%$ (24 data points) or $1.9 \% \leq \phi-\phi_{\mathrm{g}} \leq 23 \%$ (42 data points). Dotted black lines highlight the respective slope of power law fits with an indication of the value of the scaling exponent $\beta$ in the fast compression regime. Full blue lines of different colors are the best power law fits of $\left(\varepsilon_{c}, \phi-\phi_{g}\right)$ data at a loading speed $d h / d t=10 \mu \mathrm{m} / \mathrm{s}, 1 \mu \mathrm{m} / \mathrm{s}$ or $0.1 \mu \mathrm{m} / \mathrm{s}$ for $0.4 \%<\phi-\phi_{\mathrm{g}}<2 \%$ and $2 \%<\phi-\phi_{\mathrm{g}}<23 \%$, respectively.

Considering a nearly incompressible hydrogel at a fast compression speed $d h / d t \geq 100 \mu \mathrm{m} / \mathrm{s}$ (Fig. 11), the critical strain $\varepsilon_{c}$ keeps a somewhat constant value $\varepsilon_{c}=(4.7 \pm 0.54) \%$ in the linear enthalpic elastic regime $\left(\phi-\phi_{\mathrm{g}}<2 \%\right)$ and decreases as $\left(\phi-\phi_{g}\right)^{-0.45}$ at higher fiber volume fraction $\phi-\phi_{\mathrm{g}}>2 \%$ (Fig.13). The problem now is to specify the origin of the non linear transition, in relation with either the dynamics of water permeation or the reorganization of the network under compression.

In such a context, the compressive strain dependence of the storage modulus in the linear shear regime can lead to additional information. For this purpose, the equilibrium storage modulus $G^{\prime *}(\varepsilon)$ and loss modulus $G^{\prime *}(\varepsilon)$ was determined in the linear shear regime (shear strain amplitude $\gamma=210^{-2} \%$ ) when superimposing a compressive strain increasing by successive steps $d \varepsilon$ from $0.5 \%$ up to $40 \%$ and after holding each compressive strain step for 1 hour (section 2.4). Surprisingly, the dimensionless storage modulus $\mathrm{G}^{\prime *}(\varepsilon) / \mathrm{G}_{\mathrm{o}}{ }^{\prime *}(\varepsilon=0)$ remains constant up to a few percent compressive strain and then decreases to reach a plateau value $G^{\prime *} / G_{o}{ }^{\prime *} \approx 0.5$ above a compressive strain of $30 \%$ (Fig. 14). The low amplitude $\gamma=0.02 \%$ of the cyclic shear strain and the almost constancy of the dimensionless loss modulus $\mathrm{G}^{* *}(\varepsilon) / \mathrm{G}_{\mathrm{o}}{ }^{\prime *}(\varepsilon=0) \approx 1$ over the whole compressive strain range $0.5 \%<\varepsilon<40 \%$ (Fig. 14) excludes any slip phenomenon that could suggest an artifact. Since the equilibrium storage modulus in the linear shear regime is representative of the elastic properties of the microstructure, the compression-softening behavior of the hydrogel likely results from an early reorganization of the network. Interestingly, time-resolved measurements of the elasticity of agar hydrogels left to dry very slowly at a constant temperature in a rheometer under zero controlled normal force (ZNF protocol) revealed a very similar $50 \%$ drop in the linear storage modulus for a $30 \%$ water loss from the hydrogel (Mao et al. $2017 \mathrm{c}$ ). At that time, the drying-induced softening behaviour of agar gels was attributed to strong microstructural changes without further details. 


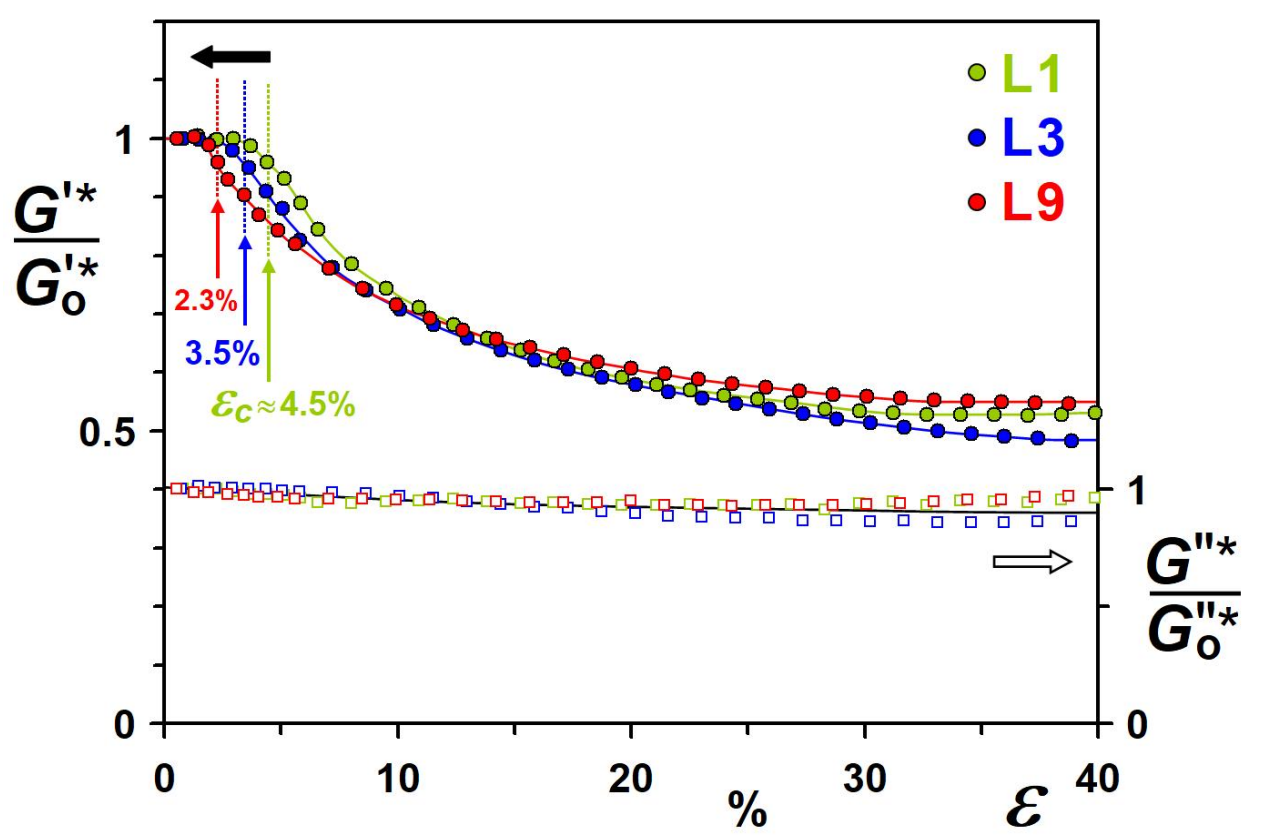

Fig. 14 Non dimensionless equilibrium storage modulus $\mathrm{G}^{\prime *} / \mathrm{G}_{\mathrm{o}}{ }^{\prime *}(\varepsilon=0)$ (left axis) and equilibrium loss modulus $\mathrm{G}^{\prime *} / \mathrm{G}_{\mathrm{o}}{ }^{* *}(\varepsilon=0)$ (right axis) of L1 (full red circles), L3 (full blue circles) and L9 (full green circles) hydrogels versus the compressive strain $\varepsilon$. SAOS experiments were performed in the linear shear regime with a cyclic shear strain of low oscillatory amplitude $\gamma=210^{-2} \%$. Vertical arrows and dotted lines indicate the critical compressive strain $\varepsilon_{c}$ determined from fast compression experiments at the onset of the non linear regime. $G_{O}{ }^{\prime *} \approx 9.8 \mathrm{kPa}$ and $G_{O}{ }^{\prime *} \approx 0.18 \mathrm{kPa}$ for $\mathrm{L} 1$ hydrogel $(c \approx 0.99 \mathrm{wt} \%), G_{O}{ }^{\prime *} \approx 112 \mathrm{kPa}$ and $G_{o}{ }^{\prime *} \approx 1.93 \mathrm{kPa}$ for L3 hydrogel $(c \approx 2.9 \mathrm{wt} \%), G_{o}{ }^{* *} \approx 552 \mathrm{kPa}$ and $G_{o}{ }^{* *} \approx 9.9 \mathrm{kPa}$ for L9 hydrogel $(c \approx 8.3 \mathrm{wt} \%)$.

As mentioned above, the normal stress - strain response of agarose hydrogels under slow compression (Fig. 7b) displays similarities to that of cellular solids such as cork (Gibson et al. 1981) or open-cell foams (Ashby 2005 ) with a linear regime and a plateau stress $\sigma^{*}$ associated with a progressive local cell collapse by buckling deformation (Gibson 2005). Rochas et al. (1996) mentioned a first order phase transition upon the deswelling of agarose hydrogels from an isotropic state to a strongly birefringent anisotropic state which could result from the buckling of the semiflexible network. More recent works reported a reversible compression-softening of biopolymer gels as a result of the elastic buckling of individual filaments that no longer contribute to the elasticity of the network (Chaudhuri et al. 2007; Kim et al. 2014, van Oosten et al. 2016, Bouzid et Del Gado 2018). Very surprisingly, fibrin networks under compression experience a 50\% stress softening at compressive strain $30 \%<\varepsilon<60 \%$ (Kim et al. 2014) almost identical to that observed for agarose hydrogels (Fig. 14). A rheometer-coupled fluorescent confocal microscope further highlights the bending and buckling of fibrin fibers resulting in a compression-softening of the network at a compressive strain of only a few percent (Kim et al. 2014).

The onset of the compression-softening of agarose hydrogels occurs above a compressive strain slightly less but close to the critical strain $\varepsilon_{c}(\phi)$ determined from fast compression experiments (Fig. 10a and Fig. 14). The transition to the non linear regime thus likely results from the buckling-induced softening of the network under compressive strain. A stiff polymer of radius $r$ with free or clamped ends undergoes a buckling instability above the Euler force threshold $F_{b} \propto E_{m} r^{4} / L^{2}$ scaling as the elastic modulus $E_{m}$ of the material and the inverse square length $L$ of the rigid strand (Gibson 2005; Baczynski K et al. 2007) whereas such a phenomenon is not observed for flexible chains as thermal motion randomizes the coil configuration. The buckling force $F_{b}\left(\xi, l_{p}, L\right)$ of semiflexible strands of length $L$ that extend over several cross-linking length scale $\xi$ still remains difficult to establish. By assuming that the buckling instability of a dense and homogeneous network of mesh size $\xi<l_{p}<L$ occurs above a critical compressive strain $\varepsilon_{c} \propto \xi$ with $\varepsilon_{c} \propto\left(\phi-\phi_{g}\right)^{-0.45}$ in the entropic elastic regime $\left(\phi-\phi_{\mathrm{g}}>2 \%\right.$, Fig. 13), the Young modulus then obeys a scaling law 
$E \approx k T / \xi^{3} \propto\left(\phi-\phi_{g}\right)^{1.35}$ similar to that derived from fast compression experiments (Fig. 12) ${ }^{6}$. On the other hand, the buckling threshold of a sparsely connected and inhomogeneous network is mainly determined by the mechanical behavior of individual strands of persistence length $l_{p}$ less than the mesh size $\xi$ of the network. In such conditions, the critical strain $\varepsilon_{c}$ of diluted agarose hydrogels is expected to be nearly constant without dependence upon the agarose fiber volume fraction as observed experimentally in the fast compression regime ( $\varepsilon_{c}=4.7 \pm 0.54 \%$ for $\phi-\phi_{\mathrm{g}}<2 \%$, Fig. 13). A strain-induced buckling criterion therefore appears relevant to describe the onset of the non linear regime during the fast compression of a semiflexible network.

Furthermore, the critical compressive strain $\varepsilon_{c}$ gradually decreases with the loading speed $d h / d t$ in the slow compression regime regardless of the agarose concentration (Fig. 13). The early transition to the non linear regime under slow compression can be due either to the progressive release of water or to the creep-induced aging of the strands that can buckle at a lower compressive strain on longer timescales (Cocks and Ashby 2000). Under very slow compression $(d h / d t=0.1 \mu \mathrm{m} / \mathrm{s})$, an inverted bell-shaped curve is further observed for the concentration dependence of the critical compressive strain $\varepsilon_{c}$ (Fig. 13). Both the buckling-induced aging of the network and the poroelasticity of the hydrogel might explain such an unexpected behaviour as discussed in the last paragraph.

\subsection{Poroviscoelasticity of agarose hydrogels}

The time relaxation of the normal stress $\sigma(t)$ was monitored just after a fast compression of the agarose hydrogel in a period of a few seconds $(d h / d t=100 \mu \mathrm{m} / \mathrm{s}$ ) and then subsequent holding of a low compressive strain $\varepsilon \approx 2 \%$ for 30 min to minimize at best non linear effects (Fig. 15).

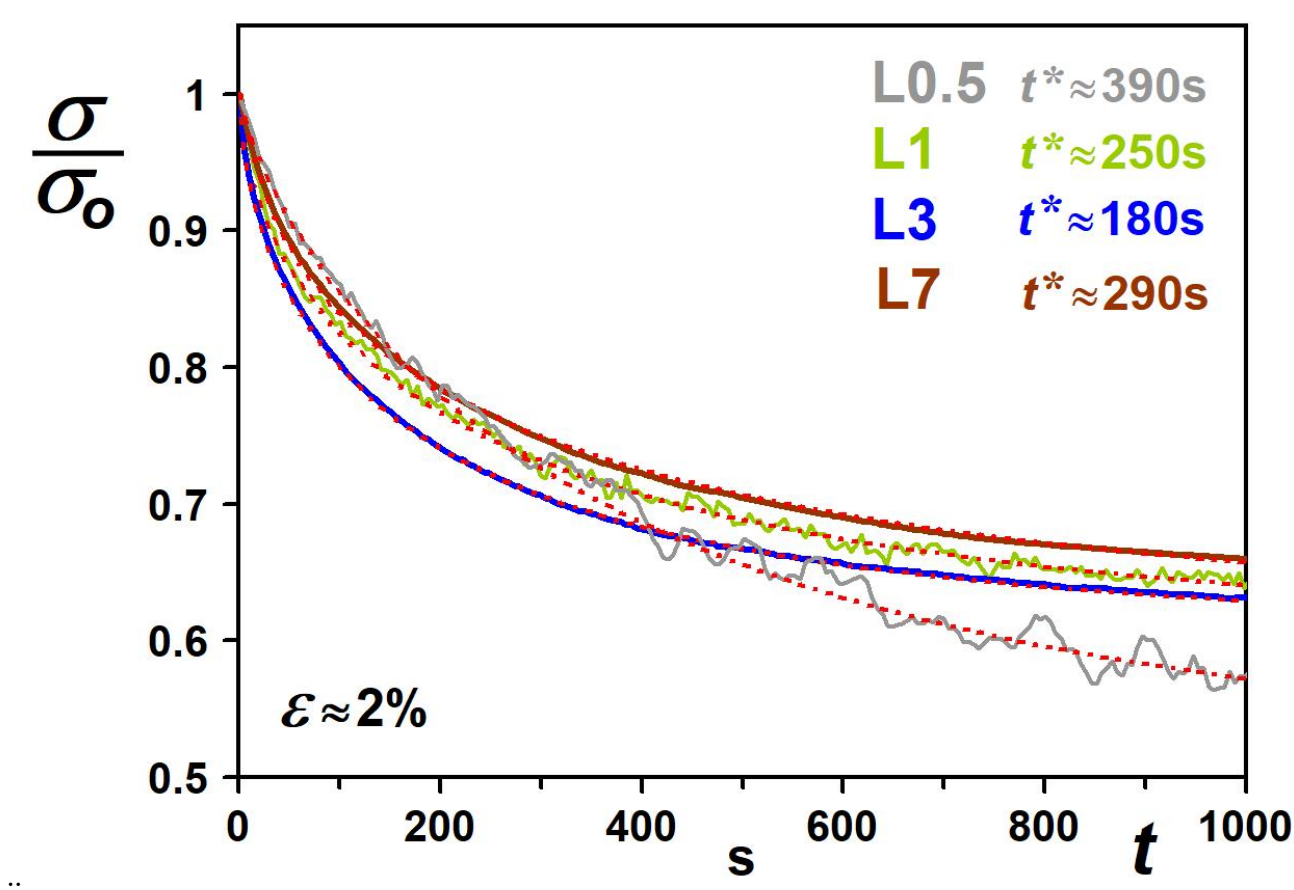

Fig. 15 Time relaxation of the dimensionless normal force $\sigma(t) / \sigma_{o}$ for L05 (grey curve), L1 (green curve), L3 (blue curve) and L9 (brown curve) hydrogels when applying a fast $2 \%$ strain ramp at a loading speed $d h / d t=100 \mu \mathrm{m} / \mathrm{s}$ and holding a static compressive strain $\varepsilon=2 \%$. Dotted red lines correspond to empirical stretched exponential functions fitted to $\left(\sigma / \sigma_{o}, t\right)$ data with the parameter values shown in Table 3. Setexam agarose hydrogels with a polymer mass concentration $c \approx 0.5 \mathrm{wt} \%$ ( L 0.5 ), $c \approx 0.99 \mathrm{wt} \%$ (L1), $c \approx 2.9 \mathrm{wt} \%$ (L3) and $c \approx 6.5 \mathrm{wt} \%$ ( L 7 ).

${ }^{6}$ The typical mesh size of concentrated agarose hydrogels scales as $\xi \propto \varepsilon_{c} \propto\left(\phi-\phi_{g}\right)^{-0.45}$ with a scaling exponent $\lambda \approx-0.45$ less than the expected value $\lambda=-0.5$ for a sparse semiflexible network since the radius $r$ of strands is no longer small enough compared to the mean diameter $\xi$ of pores. 
The dimensionless load relaxation $F(t)=\sigma(t) / \sigma_{o}$ was analyzed using an empirical stretched exponential function (Eq. 2 in section 2.4) to determine the reduced equilibrium stress $\sigma^{*} / \sigma_{o}$ and the characteristic permeation time $t^{*}$ (Table 3 ).

On long timescales, the fluid pressure becomes equal to the hydrostatic pressure and the network alone supports the equilibrium normal stress $\sigma^{*}$. For a purely poroelastic hydrogel, the expression of the reduced equilibrium stress $\sigma^{*} / \sigma_{o}$ in the linear regime can be derived from the Young modulus $E=2 G_{o}(1+v)$ :

$\sigma^{*} / \sigma_{o}=(2 / 3)\left(1+v^{*}\right)$

where $\sigma_{o}=3 G_{o} \varepsilon$ is the instantaneous compressive stress in the limit $d \varepsilon / d t \rightarrow \infty$ and $v^{*}$ the drained Poisson's ratio of the poroelastic material.

\begin{tabular}{|c|c|c|c|c|c|c|c|}
\cline { 2 - 7 } \multicolumn{1}{c|}{} & $\begin{array}{c}c \\
(\mathrm{wt} \mathrm{\% )}\end{array}$ & $\begin{array}{c}\sigma_{o} \\
(\mathrm{kPa})\end{array}$ & $\sigma^{*} / \sigma_{o}$ & $\begin{array}{c}t^{*} \\
(\mathrm{~min})\end{array}$ & $s$ & $\begin{array}{c}v^{*} \text { derived } \\
\text { from (4) }\end{array}$ & $\begin{array}{c}v^{*} \text { derived from } \\
\text { gel observations }\end{array}$ \\
\hline L 0.5 & 0.48 & 0.18 & 0.48 & 6.5 & 0.7 & -0.28 & $0.08 \pm 0.045$ \\
\hline L 1 & 0.99 & 0.54 & 0.6 & 4.2 & 0.6 & -0.1 & $0.13 \pm 0.04$ \\
\hline L 3 & 2.9 & 7.2 & 0.605 & 3 & 0.6 & -0.09 & $0.145 \pm 0.04$ \\
\hline L 7 & 6.5 & 24.6 & 0.61 & 4.8 & 0.6 & -0.085 & $0.2 \pm 0.04$ \\
\hline
\end{tabular}

Table 3 Maximum normal stress $\sigma_{o}(t=0)$, dimensionless equilibrium normal stress $\sigma^{*} / \sigma_{o}$, characteristic permeation time $t^{*}$ and drained Poisson's ratio $v^{*}$ of L $0.5, \mathrm{~L} 1, \mathrm{~L} 3$ or L 7 agarose hydrogels when holding a fast $2 \%$ compressive strain ramp and monitoring the time variations of the normal force $F(t)$ for $30 \mathrm{~min}$. An empirical stretched exponential function fitted to data $\sigma(t) / \sigma_{o}$ yields the parameter values $\sigma * / \sigma_{o}, t^{*}$ and $s$ (Eq. 2). The drained Poisson's ratio $v^{*}$ is either determined from Eq. 4 or from the observation of the equilibrium hydrogel under a static compressive strain $\varepsilon=4 \%$ by using the spatiotemporal analysis (open red squares symbols in Fig. 11).

The characteristic permeation time $t^{*}$ derived from the temporal relaxation of the normal stress take values from $3 \mathrm{~min}$ up to $6.5 \mathrm{~min}$ at agarose concentrations $0.5 \mathrm{wt} \%<c<6.5 \mathrm{wt} \%$ (Fig. 15 and Table 3) in agreement with previously reported values for centimeter-sized agarose hydrogels (Strange et al. 2013, Kaneda and Iwasaki 2015). The fairly rapid water drainage from a macroscopic agarose sample is due to the stiffness of semiflexible biopolymer hydrogels and to the high hydraulic permeability of the network ${ }^{7}$.

The drained Poisson's ratio $v^{*}$ determined from Eq.4 slightly increases from -0.28 up to -0.085 with the agarose concentration $0.5 \mathrm{wt} \%<c<6.5 \mathrm{wt} \%$ (Table 3). On the other hand, the direct observation of the hydrogel volume changes over long time scale using the spatiotemporal analysis indicates higher values of the drained Poisson's ratio $v^{*}$ increasing from 0.08 up to 0.2 over the same concentration range $0.5 \mathrm{wt} \%<c<6.5 \mathrm{wt} \%$ when holding a static compressive strain $\varepsilon=4 \%$ (Fig. 11 and Table 3 ) ${ }^{8}$. The viscoelasticity of agarose hydrogels likely accounts for the low negative wrong value of the drained Poisson ratio $v^{*} \approx-0.1$ derived from Eq.4 compared to the values $0.13<v^{*}<0.2$ determined from the imagery observation of the drained gel at polymer concentration $1 \mathrm{wt} \%<\mathrm{c}<6.5 \mathrm{wt} \%$. Poroelasticity and viscoelasticity coexist in agarose hydrogels with a retarded shear elastic response $\left(G_{2}+G_{3}\right) / G_{o}$ in the range from $15 \%$ down to $10 \%$ for agarose concentrations $1 \mathrm{wt} \%<c<6.5 \mathrm{wt} \%$ (Fig. 19 in appendix 5.4 and Table 4). An additional drop $-15 \%<-\delta\left(\sigma^{*} / \sigma_{o}\right)<-10 \%$ in the equilibrium reduced normal stress indeed results in an extra decrease

${ }^{7}$ The forced permeation time $t^{*} \propto \eta R^{2} /(E k)$ of a solvent through a semiflexible hydrogel under compression scales as the square of the cylinder radius $R$ and as the inverse of the elastic modulus $E$ of the soft material where $k \propto \xi^{2}$ is the hydraulic permeability of the network and $\eta$ the viscosity of the solvent (Doi 2009).

${ }^{8}$ A minimum compressive strain $\varepsilon=4 \%$ is required for an accurate determination of the drained Poisson's ratio using the spatiotemporal method. 
$-0.23<\delta v^{*}=-(3 / 2) \delta\left(\sigma^{*} / \sigma_{\mathrm{o}}\right)<-0.15$ in $v^{*}$ calculated from Eq.4 which approximately accounts for the observed deviation (two last columns in Table 3 ).

The compression-softening of the hydrogel further contributes to lower the equilibrium stress $\sigma^{*}$ when the compressive strain exceeds the critical strain $\varepsilon_{c}$ and then Eq.4 leads to strongly negative wrong values of the drained Poisson's ratio. The higher retarded elasticity $\left(\mathrm{G}_{2}+\mathrm{G}_{3}\right) / \mathrm{G}_{\mathrm{o}} \approx 48 \%$ of the L0.5 agarose hydrogel under a weak compressive strain $\varepsilon=4 \%$ (Fig. 19 in appendix 5.4 and Table 4) is likely associated with a buckling-induced aging of the soft network on long timescale thus resulting in the strongly negative wrong value $v^{*} \approx-0.28$ of the drained Poisson's ratio derived from Eq.4 (Table 3). Indeed, negative values of the Poisson's ratio $v^{*}$ from -0.5 down to -0.7 are incorrectly reported for agarose and biopolymer hydrogels in the literature (Strange et al. 2013, Caccavo et al. 2017) as the too large compressive strain $\varepsilon \approx 10 \%>\varepsilon_{\mathrm{c}}$ usually considered and the strain holding period of several hours cause significant non linear effects and a consequent additional drop in the equilibrium normal stress $\sigma^{*}$.

\begin{tabular}{|c|c|c|c|c|c|c|c|}
\cline { 2 - 8 } \multicolumn{1}{c|}{} & $\begin{array}{c}c \\
(\mathrm{wt} \%)\end{array}$ & $\begin{array}{c}G_{o} \\
(\mathrm{kPa})\end{array}$ & $\begin{array}{c}G_{2} \\
(\mathrm{kPa})\end{array}$ & $\begin{array}{c}t_{2} \\
(\mathrm{sec})\end{array}$ & $\begin{array}{c}G_{3} \\
(\mathrm{kPa})\end{array}$ & $\begin{array}{c}t_{3} \\
(\mathrm{~min})\end{array}$ & $\begin{array}{c}\left(G_{2}+G_{3}\right) / G_{o} \\
(\%)\end{array}$ \\
\hline L 0.5 & 0.48 & 1.55 & 0.13 & 110 & 0.61 & 30 & 48 \\
\hline L 1 & 0.99 & 9.43 & 0.45 & 83 & 1 & 30 & 15.4 \\
\hline L 3 & 2.9 & 105 & 4.9 & 35 & 11.7 & 30 & 15.8 \\
\hline L 7 & 6.5 & 366 & 19 & 16 & 15 & 30 & 10 \\
\hline
\end{tabular}

Table 4 Instantaneous elastic shear modulus $G_{o}$, viscoelastic shear moduli $\left(G_{2}, G_{3}\right)$ respectively at short and long timescales $\left(t_{2}, t_{3}\right)$ and retarded elasticity $\left(G_{2}+G_{3}\right) / G_{o}$ of quasi-equilibrium L $0.5, \mathrm{~L} 1, \mathrm{~L} 3$ and L 7 agarose hydrogels weakly compressed in water when superimposing a low static shear strain $\gamma$ in the linear regime. Before shearing the hydrogel, a fast $4 \%$ compressive strain ramp is imposed at a loading speed $d h / d t=100 \mu \mathrm{m} / \mathrm{s}$ and the static compressive strain $\varepsilon=4 \%$ is hold for $15 \mathrm{~min}$. A second order generalized Maxwell model (Eq. 1) fitted to normal stress data $\tau(t)$ gives the parameter values $G_{o}, G_{2}, t_{2}, G_{3}, t_{3}$.

The imagery observation of the agarose hydrogel under weak unconfined compression thus appears as a more reliable method for correctly and unambiguously measuring the drained Poisson's ratio $v^{*}$ of centimeter-sized samples as the permeation time $t^{*}$ of a few minutes is of the same order as the viscoelastic characteristic time (Fig. 15 and Fig. 19 in appendix 5.4). Microindentation methods probing the hydrogel at smaller length scales or unconfined compression of enough large samples represent a way to speed up or slow down water exudation in order to separate poroelasticity and viscoelasticity (Strange et al. 2013). However, the geometric confinement of the hydrogel under a spherical indenter slows water migration and makes more complex the expression of the equilibrium normal stress (Chan et al. 2012; Delavoipière et al. 1016).

Finally, the delayed and higher apparent viscoelasticity of softer hydrogels likely due to the buckling-induced aging of the network in the enthalpic elastic regime (sample L 0.5 in Fig. 19) results in an increase of both the permeation time $t^{*}(\phi)$ (sample L 0.5 in Fig. 15) and the critical strain $\varepsilon_{c}$ under very slow compression (Fig. 13). On the other hand, stiffer hydrogels exhibit a much lower viscoelasticity in the entropic elastic regime (samples L1, L 3 and L 7 in Fig. 19) but the slower rate of water exudation from the dense network contribute to an increase in both the permeation time $t^{*}(\phi)$ (samples L1, L 3 and L 7 in Fig. 15) and the critical strain $\varepsilon_{c}$ under very slow compression (Fig. 13). Both the buckling-induced aging of sparsely connected networks and the dominant poroelasticity of denser hydrogels can thus account for the unexpected inverted bell-shaped curve for the concentration dependence of the critical compressive strain $\varepsilon_{c}$ under very slow compression (loading speed $\mathrm{dh} / \mathrm{dt}=0.1 \mu \mathrm{m} / \mathrm{s}$, full light blue circles in Fig. 13).

\section{Summary and conclusion}

One of the original contributions of the present work is the measurement of the Poisson's ratio $v$ of agarose hydrogels in the linear regime over a wide range of loading speed and polymer concentration. For this purpose, carrefully centimeter-sized pre-moulded hydrogels were submitted to a compressive strain ramp between impermeable and frictionless glass plates. Slippery conditions avoid a barrelling type deformation of the hydrogel in the linear compressive regime as observed with the traditional clamping of the gel to the top and bottom surfaces of plates (Kaneda and Iwasaki 2015). The linear elastic behavior of agarose hydrogels was 
shown to display two types of time response. The hydrogel remains nearly incompressible for a few seconds when the strain rate $d \varepsilon / d t$ of the compressive ramp is greater than $0.5 \% \mathrm{~s}^{-1}$. On the other hand, water exudation from the porous material occurs very early during a slow strain ramp $\left(d \varepsilon / d t<0.5 \% \mathrm{~s}^{-1}\right)$ and the hydrogel appears as a softer compressible material in the linear regime as demonstrated by the lower values of both the Young modulus and the Poisson's ratio. In the case of a very slow compression ramp ( $d \varepsilon / d t \approx 710^{-4} \% \mathrm{~s}^{-1}$ ), the hydrogel is in a near equilibrium state with values of the Poisson's ratio $v$ in the linear regime close to that of the drained Poisson's ratio $v^{*}$ on long timescale (Fig. 11). In the linear regime, the elastic modulus $E /[2(1+v)]$ takes values quite close to those of the shear modulus $G_{o}$ whatever the compressive strain rate of the hydrogel as predicted by the linear poroelastic theory. Such a result confirms the weak viscoelasticity of fibrillar hydrogels and the significant influence of the deformation process of the waterlooged material upon the elastic response not just determined by the mechanical properties of the network.

The fiber volume fraction dependence of both the Young modulus and the shear elastic modulus reveals a hydrogel deformation mode that is either dominated by the bending of semiflexible strands (non affine enthalpic regime) at low agarose concentrations or by the stretching of the network (affine entropic regime) at higher polymer concentrations as reported earlier by Jones and Marques (1990) and Ramzi et al. (1998). In the enthalpic elastic regime, the bending of strands of persistence length less than the mesh size of the sparsely connected fibrillar network is easier than the stretching of polymer chains which gives rise to floppy modes and non affine deformations as observed in fibrin clots (Kim et al. 2014) and fiber network models (Wen et al. 2012, Huisman and Lubensky 2011). The deformation of the network evolves toward a stretching dominated affine regime when increasing the crosslink density and displays an entropic elastic behaviour as the microstructure of highly concentrated agarose hydrogels is currently viewed as rigid strands connected by flexible junctions (Djabourov et al. 2013). In the very high concentration regime $c>10 \mathrm{wt} \%$, the present work demonstrates the occurrence of a third regime with a linear concentration dependence of elastic moduli as all the strands contribute to the elasticity of the strongly connected network.

Another original contribution of the work is the highlighting of the mechanism at the origin of the transition to the non linear regime when applying relatively small compressive strains. The buckling-induced softening of the fibrillar network indeed appears relevant to describe the onset of the non linear regime during the fast compression of agarose hydrogels. Buckled strands no longer contribute to the elasticity of the network and the deformation is nearly reversible as the strands unbuckle after removal of the load. In the entropic elastic regime, the length of connected strands is less than the persistence length and the compression-softening of the hydrogel under fast compression occurs above a critical strain $\varepsilon_{c}$ scaling as the mesh size $\xi$ of the network. In the enthalpic elastic regime, the buckling instability of semiflexible strands in a weakly connected network requires a critical compressive strain of about $5 \%$ without significant dependence on the agarose concentration at $c<2 \mathrm{wt} \%$. Interestingly, the present work reports a compression-softening of agarose hydrogels with a very low dependence on network topology and strand connectivity while numerical simulations only predict a strainsoftening of semiflexible gels for sparsely connected networks (Bouzid and Del Gado 2018).

The lower values of the critical compressive strain $\varepsilon_{c}$ under slow compression likely indicates a bucklinginduced aging of the strands. Within such a context, both the retarded buckling of the network and the poroviscoelasticity of agarose hydrogels contribute to the time relaxation of the normal stress. The longer permeation time $t^{*}$ of diluted agarose hydrogels results from the higher viscoelasticity of softer materials in relation with the nearly reversible buckling-induced aging of the sparsely connected network. On the other hand, the dominant poroelasticity of highly connected networks in the entropic elastic regime is responsible for the increasing permeation time of concentrated agarose hydrogels. As a consequence of the buckling of the network, the drained Poisson's ratio $v^{*}$ determined from the poroelastic equilibrium normal stress $\sigma^{*}$ can take wrong negative values when a too large compressive strain or a too long strain holding period cause a non linear compression-softening of the fibrillar hydrogel.

The buckling of semiflexible networks at small compressive strains thus induces a rich and complex mechanical behaviour of biopolymer systems and cellular materials (Foucard et al. 2015). The buckling instability in swelling or shrinking hydrogels usually occurs in the poorly connected regions near the free surface in the form of wrinkles and propagate later within the drained network since a crosslinked microsctructure becomes unstable when the volume change is too large (Doi 2009). A syneresis phenomenon has been reported for gelatin, polysaccharides and agar hydrogels consisting in a very slow shrinkage of the network and a progressive exudation of solvent (Kunitz 1928; Matsuhashi 1990; Divoux, Mao and Snabre 2015). The bucklingaging of sparse and inhomogeneous networks likely promotes such a slow spontaneous shrinking of the hydrogel usually associated with the thermally-induced formation of weak-bonds. Sparsely connected networks indeed show greater syneresis with a magnitude decreasing as the inverse square of the polymer concentration in agar 
hydrogels (Stanley 2006). In another context, the deformation mode of a fibrillar network further determines the asymmetric response of biopolymer hydrogels under a compression - tension cycle since the bending and buckling of fibers under compression is easier than the stretching of polymer chains during extension (Section 5.2). To our knowledge, the present contribution is finally among the first to monitor both the mechanical response and the volume changes in biopolymer hydrogels under compression over a wide range of loading speed and polymer concentration to highlight the buckling-induced transition to the non linear regime and determine unambiguously the Poisson's ratio on short and long timescales

\section{Appendix}

\subsection{Mean pore diameter of agarose hydrogels}

Cryo-SEM micrographs of agarose hydrogels show random fibrillar networks with a typical mean pore diameter $d$ decreasing with the mass concentration of the polymer from a micrometer size at $c \approx 0.3 \mathrm{wt} \%$ down to a few hundreds of nanometers at $c \approx 3 \mathrm{wt} \%$ (Fig. 2). The pore size distribution is broad with a factor of about 10 between the mean smaller diameter $d_{\min }$ and the mean larger diameter $d_{\max }$ of pores in the network (full black and red circles in Fig. 16). Mechanical-scanning probe microscopy indeed gives evidence of the inhomogeneous microstructure of agarose hydrogels at low concentrations $0.5 \mathrm{wt} \%<c<2 \mathrm{wt} \%$ with a $\log$ normal distribution of the local shear modulus while a simple Gaussian distribution describes the histogram of local elastic moduli values in thermal gels made of flexible chains such as polyacrylamide (Nitta et al. 2003). As expected for a fibrillar semiflexible network with a mesh size larger than the strand diameter, the mean pore diameter of agarose hydogels scales as $\left(c-c_{g}\right)^{\lambda}$ with $\lambda \approx-0.5$ at $0.3 \mathrm{wt} \%<c<3 \mathrm{wt} \%$ whatever one considers either the larger or the smaller free spaces (Fig. 16). Note that the scaling exponent $\lambda \approx-0.5$ takes slightly more negative values when considering the mass concentration in place of the deviation concentration $c-c_{g}$ from the percolation threshold (Maaloum et al. 1998; Righetti et al. 1981). Atomic force microscopic observations from Maaloum et al. (1998) or studies of the electrophoretic mobility of latex particles in agarose hydrogels from Righetti et al. (1981) indeed lead to a similar scaling exponent $\lambda \approx-0.5$ provided that the mean pore diameter is plotted versus the deviation concentration $c-c_{g}$ from the percolation point (Fig. 16). The length scale explored in sparsely connected networks is further very sensitive to the choice of the investigation method due to the broad pore size distribution of biopolymer hydrogels.

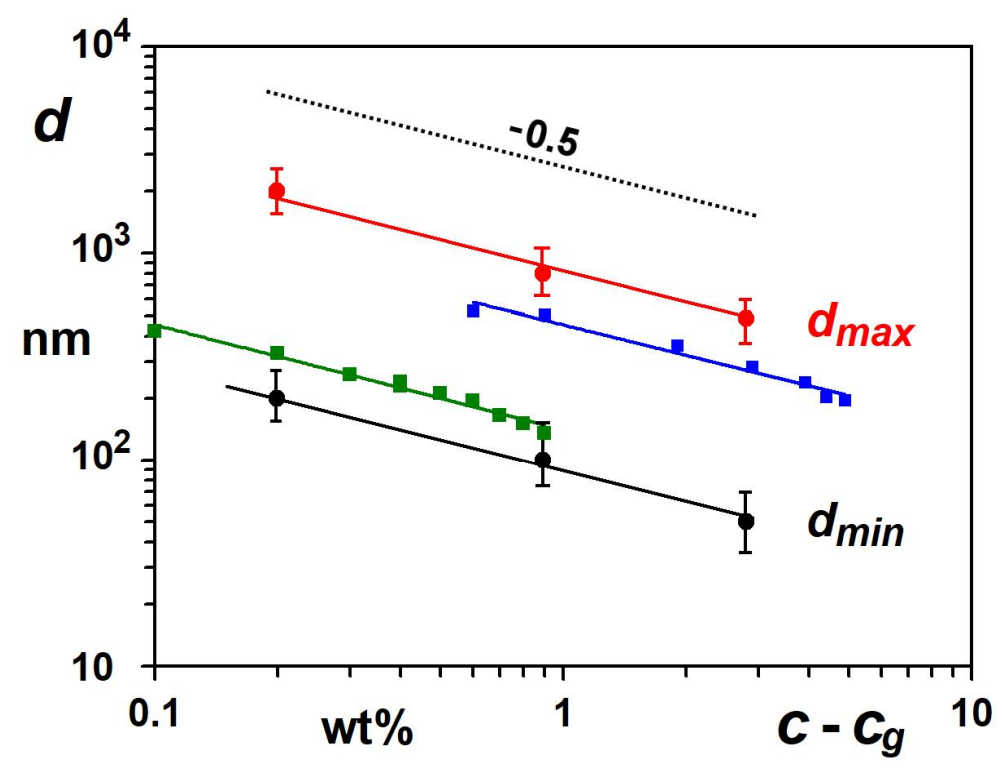

Fig. 16 Logarithmic plot of the mean pore diameter $d$ versus the agarose mass concentration $c-c_{g}$ with a gelation threshold $c_{g}=0.1 \mathrm{wt} \%$. Full black circles and red circles refer to the mean smaller diameter $d_{\min }$ and the mean larger diameter $d_{\max }$ of pores extracted from Cryo-SEM micrographs of Setexam hydrogels at polymer mass concentration $c \approx 0.3 \mathrm{wt} \% \quad(\mathrm{~L} 0.3), c \approx 0.99 \mathrm{wt} \% \quad(\mathrm{~L} 1)$ and $c \approx 2.9 \mathrm{wt} \%$ (L3). Full green squares and full blue squares respectively stand for the experimental data of Maaloum et al. (1998) and Righetti et al (1981). Full color lines are the best power law fits of $\left(d, c-c_{g}\right)$ data and the dotted black line highlights the common slope of the power law fits with an indication of the value of the scaling exponent $\lambda \approx-0.5$. 


\subsection{Compression - tension of agarose hydrogels}

The reversibility of the hydrogel response was studied by applying a compression - tension cycle at a speed $d h / d t=100 \mu \mathrm{m} / \mathrm{s}$ with a maximum compressive strain $\varepsilon_{m}$ of either $2 \%$ (black curves in Fig. 17) or $10 \%$ (red curves in Fig. 17). The stress - strain response appears as nearly reversible in the linear regime for a maximum compressive $\varepsilon_{\mathrm{m}} \approx 2 \%$ less than the critical strain $\varepsilon_{c}$ as water exudation remains negligible during a fast compression - tension cycle (black curves in Fig. 17). A more detailed examination of $\sigma(\varepsilon)$ curves nevertheless shows a weak hysteresis and a slope $(d \sigma / d \varepsilon)\left(\varepsilon=\varepsilon_{m}\right)$ less important at the end of the compressive ramp compared to the beginning of the tension ramp, especially at low agarose concentration ( L1 sample in Fig. 17a). The asymmetry observed in the response of agarose hydrogels to a compression - tension cycle in the linear regime likely results from the deformation mode of strands since it is easier to bend a fiber during compression than to stretch the fiber during extension.
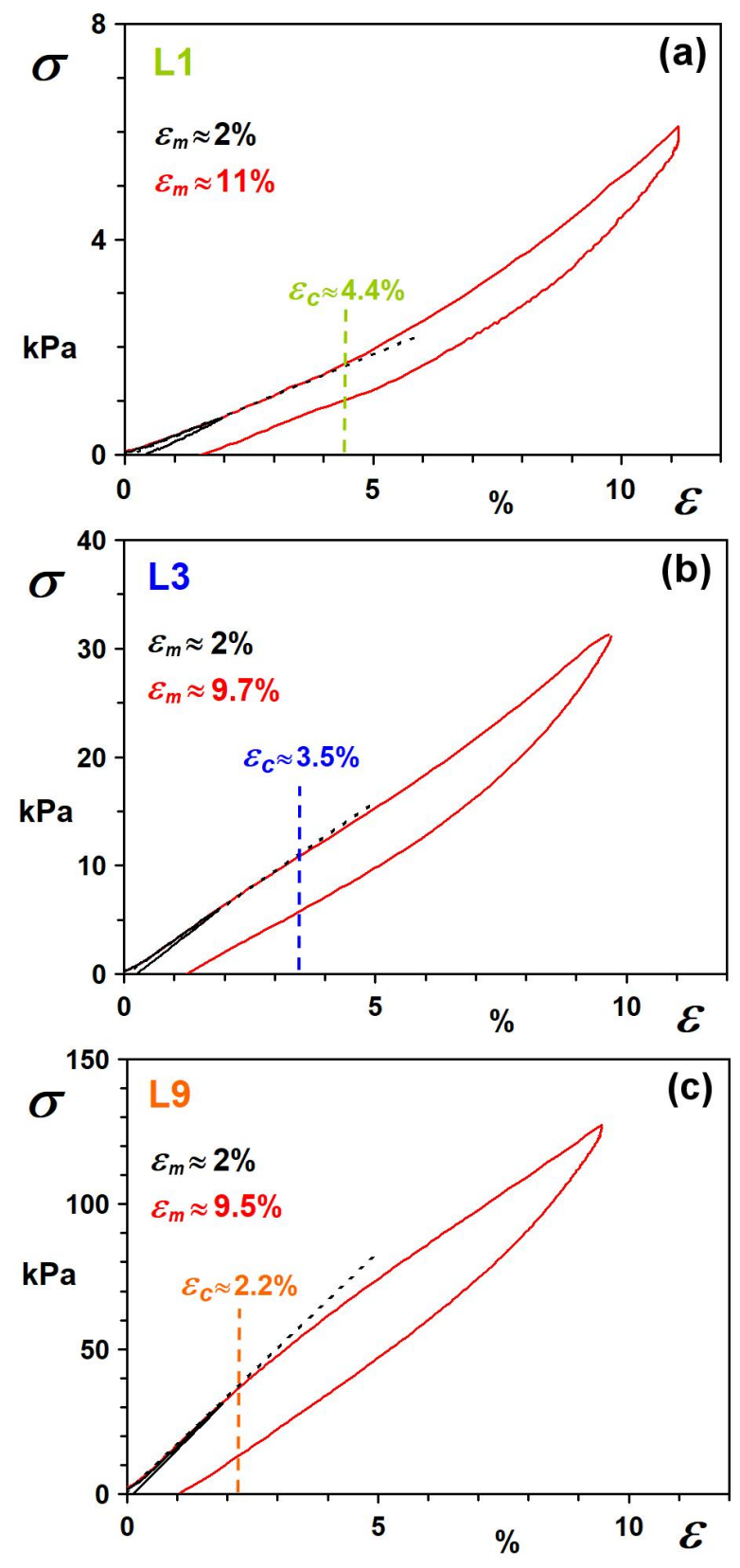

Fig. 17 Normal stress $\sigma$ versus the compressive strain $\varepsilon$ of L1 (a), L3 (b) and L9 (c) hydrogels when applying a compression - tension cycle at a loading speed $d h / d t=100 \mu \mathrm{m} / \mathrm{s}$ with a maximum amplitude $\varepsilon_{\mathrm{m}} \approx 2 \%$ (black curves) or $\varepsilon_{\mathrm{m}} \approx 10 \%$ of the strain ramp (red curves). Dashed vertical full lines show the 
critical compressive strain $\varepsilon_{c}$ delimiting the transition to the non linear regime. Setexam agarose hydrogels with polymer mass concentration $c \approx 0.99 \mathrm{wt} \%$ ( L1), $c \approx 2.9 \mathrm{wt} \%$ ( L3 ) and $c \approx 8.3 \mathrm{wt} \%$ ( L9).

As expected, the hysteresis becomes more significant in the non linear regime for a maximum compressive strain $\varepsilon_{\mathrm{m}} \approx 10 \%>\varepsilon_{\mathrm{c}}$ (full red lines in Fig. 17) as the unbuckling of strands and the breaking of some weak bonds require longer timescales during the extension phase. The degree of hysteresis increases somewhat with the polymer concentration (Fig. 17) and the gel cylinder almost recovers the initial height $h_{o} \approx 14 \mathrm{~mm}$ after a time period from a few minutes to a few hours when removing the load which confirms the dominant elastic behavior of agarose hydrogels. The compression - softening of the fibrillar network thus appears as nearly reversible even for very large compressive strain at very low loading speed as long as the hydrogel remains intact without any stress-induced microfractures. A second compression - tension cycle is necessary to observe a fully reversible deformation of the agarose hydrogel (data not shown) likely as the result of the formation of some extra irreversible bonds between buckled strands during the first compression ramp (paper in preparation).

\subsection{Agarose molecular weight and elasticity of agarose hydrogels}

Compression experiments were performed using the Brookfield texture analyzer and agarose hydrogels prepared at different concentrations $0.5 \mathrm{wt} \% \leq c \leq 9 \mathrm{wt} \%$ with two different powders supplied either by Setexam or Sigma (section 2.1). The stress - strain curves of Sigma and Setexam agarose hydrogels considered as incompressible when applying a fast $15 \%$ strain ramp at a loading speed $d h / d t=100 \mu \mathrm{m} / \mathrm{s}$ exhibit similar features (Fig. 10a and Fig. 18a). As expected, the agarose average molecular weight weakly influences the scaling exponents $\beta \approx 2.15 \pm 0.05$ or $\beta \approx 1.36-1.41$ representative of either the enthalpic or the entropic elasticity (Fig. 18b). However, Fig. 18b gives evidence of higher values of the Young modulus $E_{o}$ of Sigma agarose hydrogels $\left(M_{w} \approx 3.0510^{5} \mathrm{~g} / \mathrm{mol}\right)$ compared to that of Setexam agarose hydrogels ( $M_{w} \approx 1.8810^{5} \mathrm{~g} / \mathrm{mol}$ ) regardless of the agarose concentration. The power law fits of the fiber volume fraction dependence of the Young modulus $E_{o}\left(\phi-\phi_{g}\right)$ of Sigma and agarose hydrogels in the enthalpic and entropic elastic regimes respectively give $E_{o} \approx M_{w}^{0.8}$. The Young modulus of biopolymer fibrillar networks is usually reported to scale as $E_{o} \approx M_{w}^{2}$ for lower average molecular weigth $310^{4} \mathrm{~g} / \mathrm{mol}<M_{w}<710^{4} \mathrm{~g} / \mathrm{mol}$ (Eldridge and Ferry 1954). The dependence on $M_{w}$ of the elastic modulus of agarose hydrogels is expected to be lower for higher average molecular weight and higher polymer concentrations as the number of dangling ends not involved in the elasticity of the network becomes less (Normand et al. 2000). 

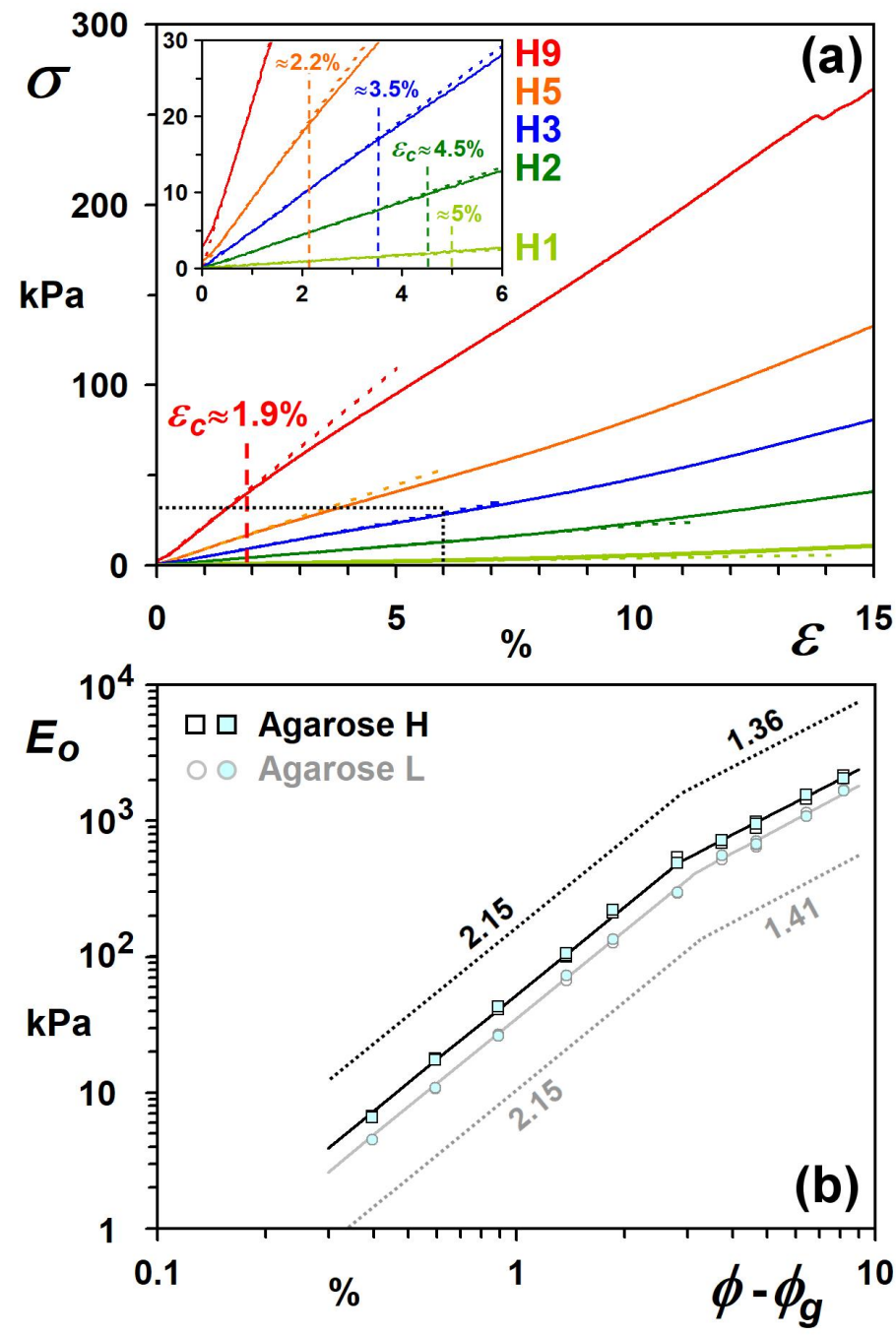

Fig. 18 (a) Normal stress $\sigma$ versus the compressive strain $\varepsilon$ for $\mathrm{H} \alpha$ hydrogels in water when applying a $15 \%$ strain ramp at a loading speed $d h / d t=100 \mu \mathrm{m} / \mathrm{s}$ in the Brookfield texture analyzer. The insert is a zoom in the low strain region where dotted lines are best linear fits of $\sigma(\varepsilon)$ data in the limit of low strain $\varepsilon<\varepsilon_{c}$ and dashed vertical lines show the critical compressive strain $\varepsilon_{c}$. (b) Semi logarithmic plot of the Young modulus $E_{o}$ of the hydrogel assumed as incompressible versus the fiber volume fraction $\phi-\phi_{g}$ with $\phi_{\mathrm{g}}=0.1 \%$ either for Sigma (square symbols) or Setexam (circle symbols) agarose cylinders rapidly compressed in air (open symbols) or in water (full blue symbols). Full black and grey lines are the best power law fits of $\left(E_{o}, \phi-\phi_{g}\right)$ data for Sigma and Setexam agarose hydrogels respectively in the concentration regime $0.4 \% \leq \phi-\phi_{\mathrm{g}} \leq 2.8 \%$ and $3.75 \% \leq \phi-\phi_{\mathrm{g}} \leq 8.2 \%$. Dotted black lines and dotted grey lines highlight the respective slopes of power law fits with an indication of the value of the elastic exponent $\beta$ either in the enthalpic or in the entropic elastic regime.

\subsection{Viscoelasticity of agarose hydrogels}

The shear stress relaxation response of quasi-equilibrium agarose hydrogels was monitored in the linear regime when applying a low compressive strain $\varepsilon \approx 2 \%$ (section 2.4). The characteristic viscoelastic time $t_{2}$ at short timescale decreases from $2 \mathrm{~min}$ down to 16 seconds when increasing the agarose concentration $0.5 \mathrm{wt} \%<c<7 \mathrm{wt} \%$ as the hydrogel becomes stiffer (Fig. 19). The retarded elastic response $\left(G_{2}+G_{3}\right) / G_{0}$ of about $10 \%$ to $15 \%$ for agarose concentration $c>1 \mathrm{wt} \%$ surprisingly takes on a much larger value $\left(G_{2}+G_{3}\right) / G_{0} \approx 48 \%$ for a diluted $0.5 \mathrm{wt} \%$ agarose hydrogel ( L 0.5 sample in Fig. 19 and Table 4). Such a sharp increase in the apparent viscoelasticity of a diluted agarose hydrogel probably results from the bucklinginduced aging of the sparsely connected network on long timescale and the gradual emergence of non linear effects. 


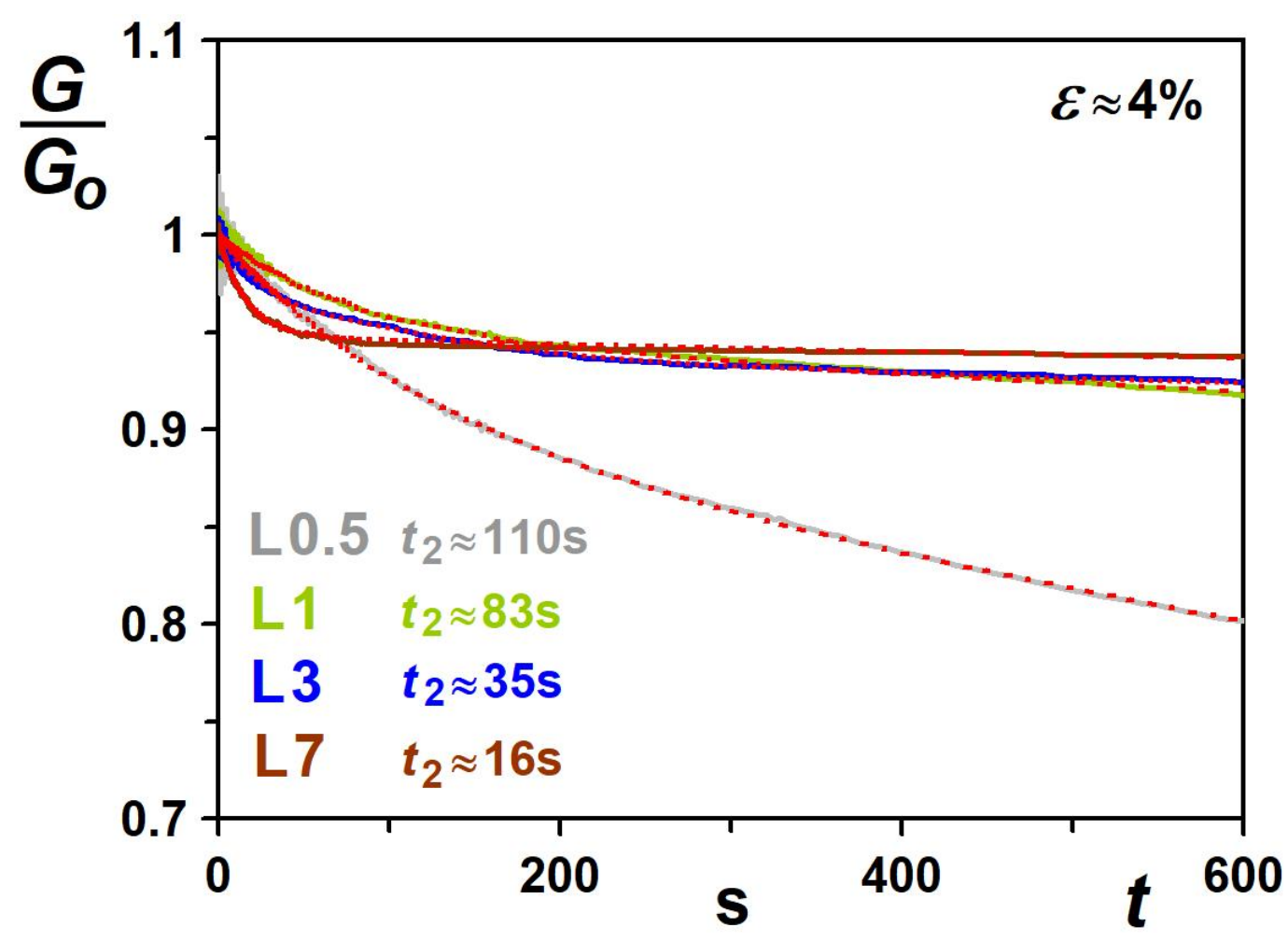

Fig. 19 Time relaxation of the dimensionless shear stress modulus $G(t) / G_{o}$ of quasi-equilibrium hydrogels L 0.5 (grey color), L1 (green color), L 3 (blue color) and L 7 (brown color) weakly compressed in water when superimposing a shear strain in the linear regime. Before the stress relaxation test, a fast $4 \%$ compressive strain ramp is imposed at a loading speed $d h / d t=100 \mu \mathrm{m} / \mathrm{s}$ and the static strain $\varepsilon=4 \%$ is hold for $15 \mathrm{~min}$. Dotted red curves correspond to a second order generalized Maxwell model (Eq. 1) fitted to data with the parameter values shown in Table 4. Setexam agarose hydrogels with a polymer mass concentration $c \approx 0.5 \mathrm{wt} \%$ ( L 0.5 ), $c \approx 0.99 \mathrm{wt} \%(\mathrm{~L} 1), c \approx 2.9 \mathrm{wt} \%$ ( L 3$)$ and $c \approx 6.5 \mathrm{wt} \%$ ( L 7 ). 


\section{References}

Armstrong CG, Lai WM, Mow VC (1984) An analysis of the unconfined compression of articular cartilage. J Biomech Eng 165:106-173. https://doi.org/10.1115/1.3138475

Arnott S, Fulmer A, Scott WE, Dea ICM, Moorhouse R, Rees DA (1974) The agarose double helix and its function in agarose gel structure. J Mol Biol 90(2):273-284. https://doi.org/10.1016/0022-2836(74)90372-6

Ashby MF (2005) The properties of foams and lattices. Philosophical Transactions of the Royal Society A 364:15-30. https://doi.org/10.1098/rsta.2005.1678

Aymard P, Martin DR, Plucknett K, Foster TJ, Norton IT (2001) Influence of thermal history on the structural and mechanical properties of agarose gels. Biopolymers 59(3):131-144. https://doi.org/10.1002/10970282(200109)59:3<131::AID-BIP1013>3.0.CO;2-8

Ayyad O, Muñoz-Rojas D, Agulló N, Borrós S, and Gómez-Romero P (2010) High-concentration compact agar gels from hydrothermal synthesis. Soft Matter 6(11):2389-2391. https://doi.org/10.1039/b926713a

Arnott S, Fulmer A, Scott WE, Dea ICM, Moorhouse R, Rees DA (1974) The agarose double helix and its function in agarose gel structure. J Mol Biol 90(2):273-284. https://doi.org/10.1016/0022-2836(74)90372-6

Baczynski K, Lipowsky R, and Kierfeld J (2007) Stretching of buckled filaments by thermal fluctuations. Phys Rev E 76:061914. https://doi.org/10.1103/PhysRevE.76.061914

Bertula K, Martikainen L, Munne P, Hietala S, Klefström J, Ikkala O, and Nonappa (2019) Strain-stiffening of agarose gels. ACS Macro Lett 8(6):670-675. https://doi.org/10.1021/acsmacrolett.9b00258

Biot M (1941) General theory of three dimensional consolidation. Journal of applied physics 12(2):155-164. https://doi.org/10.1063/1.1712886

Bouzid M, and Del Gado E (2018) Network topology in soft hydrogels: Hardening and Softening materials. Langmuir 34(3): 773-781. https://doi.org/10.1021/acs.langmuir.7b02944

Broedersz CP, Mao X, Lubensky TC, and MacKintosh FC (2011) Criticality and isostaticity in fiber networks Nature Physics 7 (12): $983-988$. https://doi.org/10.1038/nphys2127

Caccavo D, Cascone S, Poto S, Lamberti G, Barba AA (2017) Mechanics and transport phenomena in agarosebased hydrogels studied by compression-relaxation tests. Carbohydrate Polymers 176:136-144. https://doi.org/10.1016/j.carbpol.2017.03.027

Cai S, Hu Y, Zhao X, and Suo Z (2010) Poroelasticity of a covalently crosslinked alginate hydrogel under compression. Journal of Applied Physics 108:113514. https://doi.org/10.1063/1.3517146

Chan EP, Hu Y, Johnson PM, Suo Z, and Stafford CM (2012) Spherical indentation testing of poroelastic relaxations in thin hydrogel layer. Soft Matter 8(5):1492-1498. https://doi.org/10.1039/c1sm06514a

Chaudhuri O, Parekh SH, and Fletcher DA (2007) Reversible stress softening of actin networks. Nature 445(7125):295-298. https://doi.org/10.1038/nature05459

Clark AH, Ross-Murphy SB (1987) Structural and mechanical properties of biopolymer gels. In: Biopolymers. Advances in Polymer Science. Springer, Berlin Heidelberg vol 83 pp 157-192. https://doi.org/10.1007/BFb0023332

Cocks ACF, Ashby MF (2000) Creep-buckling of cellular solids. Acta Mater 48(13):3395-3400. https://doi.org/10.1016/S1359-6454(00)00139-7

Coviello T, Kajiwara K, Burchard W, Dentini M, and Crescenzi V (1986) Solution properties of xanthan. 1. Dynamic and static light scattering from native and modified xanthans in dilute solutions. Macromolecules 19(11):2826-2831. https://doi.org/10.1021/ma00165a027

de Gennes PG (1980) Scaling concepts in polymer physics. Cornell University Press, Ithaca. https://doi.org/10.1002/actp.1981.010320517 
Delaine-Smith RM, Burney S, Balkwill FR, Knight MM (2016) Experimental validation of a flat punch indentation methodology calibrated against unconfined compression tests for determination of soft tissue biomechanics. J Mech Behav Biomed Mater 60:401-415. https://doi.org/10.1016/j.jmbbm.2016.02.019

Delavoipière J, Tran Y, Verneuil E, Chateauminois A (2016) Poroelastic indentation of mechanically confined hydrogel layers. Soft Matter 12(38):8049-8058. https://doi.org/10.1039/C6SM01448H

Divoux T, Mao B, and Snabre P. (2015) Syneresis and delayed detachment of agar plates. Soft Matter 11(18) 3677-3685. https://doi.org/10.1039/c5sm00433k

Djabourov M (1991) Gelation - A review. Polymer International 25(3):135-143. https://doi.org/10.1002/pi.4990250302

Djabourov M, Clark AH, Rowlands DW, and Ross-Murphy SB (1989) Small-angle X-ray scattering characterization of agarose sols and gels. Macromolecules 22(1):180-188. https://doi.org/10.1021/ma00191a035

Djabourov M, Nishinari K, and Ross-Murphy S (2013) Helical structures from neutral biopolymers. In: Physical Gels from Biological and Synthetic Polymers. Cambridge University Press. pp 182-221. https://doi.org/10.1017/CBO9781139024136

Doi M (2009) Gel dynamics. J Phys Soc Jpn 78(5):052001. https://doi.org/10.1143/JPSJ.78.052001

Dormoy Y, and Candau S (1991) Transient electric birefringence study of highly dilute agarose solutions. Biopolymers 31(1):109-117. https://doi.org/10.1002/bip.360310110

Eldridge JE, and Ferry JD (1954) Studies of the cross-linking process in gelatin gels: III. Dependence of melting point on concentration and molecular weight. J Phys Chem 58(11):992-995. https://doi.org/10.1021/j150521a013

Feke GT, and Prins W (1974) Spinodal phase separation in a macromolecular sol $\rightarrow$ gel transition. 7(4):527-530. https://doi.org/10.1021/ma60040a022

Foord SA, and Atkins EDT (1989) New X-ray diffraction results from agarose: Extended single helix structures and implications for gelation mechanism. 28(8):1345-1365. https://doi.org/10.1002/bip.360280802

Forte AE, D'Amico F, Charalambides MN, Dini D, Williams JG (2015) Modelling and experimental characterization of the rate dependent fracture of gelatine gels. Food Hydrocolloids 46:180-190. https://doi.org/10.1016/j.foodhyd.2014.12.028

Foucard LC, Price JK, Klug WS, and Levine AJ (2015) Cooperative buckling and the nonlinear mechanics of nematic semiflexible networks. Nonlinearity 28(9):R89-R112. https://doi.org/10.1088/0951-7715/28/9/R89

Fujii T, Yano T, Kumagai H, Miyawaki O (2000) Scaling analysis on elasticity of agarose gel near the sol-gel transition temperature. Food Hydrocolloids 14(4):359-363. https://doi.org/10.1016/S0268-005X(00)00012-6

Gibson LJ (2005) Biomechanics of cellular solids. Journal of Biomechanics 38(3):377-399. https://doi.org/10.1016/j.jbiomech.2004.09.027

Gibson LJ, Easterling KE, and Ashby MF (1981) The structure and mechanics of cork. Proc R Soc Lon A377:99-117. https://doi.org/10.1098/rspa.1981.0117

Greaves GN, Greer AL, Lakes RS and Rouxel T (2011) Poisson's ratio and modern materials. Nature Materials 10(11):823-837. https://doi.org/10.1038/nmat3134

Gunasekaran S, Yoon WB (2014) Investigation of elastic modulus of xanthan and locust bean gum at different concentrations of mixture using cascade model. Journal of texture studies 45(1):80-87. https://doi.org/10.1111/jtxs. 12040

Hay JL, Wolff PJ (2001) Small correction required when applying the Hertzian contact model to instrumented indentation data. 16(5):1280-1286. https://doi.org/10.1557/JMR.2001.0179

Hayashi A, Kinoshita A, and Kuwano M (1977) Studies of the agarose gelling system by the fluorescence polarization method. I. Polym J 9(2):219-225. https://doi.org/10.1295/polymj.9.219 
$\mathrm{Hu}$ Y, Suo Z (2012) Viscoelasticity and poroelasticity in elastomeric gels. Acta Mechanica Solida Sinica 25(5):441-458. https://doi.org/10.1016/S0894-9166(12)60039-1

Hu Y, Zhao X, Vlassak JJ, and Suo Z (2010) Using indentation to characterize the poroelasticity of gels. Appl. Phys. Lett 96(12):112904. https://doi.org/10.1063/1.3370354

Huisman EM, Lubensky TC (2011) Internal stresses, normal modes, and nonaffinity in three dimensional biopolymer networks. Phys Rev Let 106:088301. https://doi.org/10.1103/PhysRevLett.106.088301

Ichinose N, Ura H (2020) Concentration dependence of the sol-gel phase behavior of agarose-water system observed by the optical bubble pressure tensiometry. Sci Rep 10:2620. https://doi.org/10.1038/s41598-02058905-8

Jansen KA, Licup AJ, Sharma A, Rens R, MacKintosh FC, and Koenderink GH (2018) The role of network architecture in collagen mechanics. Biophys J 114(11):2665-2678. https://doi.org/10.1016/j.bpj.2018.04.043

Joly-Duhamel C, Hellio D, Ajdari A, and Djabourov M (2002) All gelatin networks: 2. The master curve for elasticity. Langmuir 18(19):7158-7166. https://doi.org/10.1021/la020190m

Jones JL, and Marques CM (1990) Rigid polymer network models. J Phys France 51(11):1113-1127. https://doi.org/10.1051/jphys:0199000510110111300

Kaneda I (2018) Effects of sweeteners on the solvent transport behaviour of mechanically-constrained agarose gels. Gels 4(23):1-9. https://doi.org/10.3390/gels4010023

Kaneda I, Iwasaki S (2015) Solvent transportation behavior of mechanically constrained agarose gels. Rheol Acta 54(5):437-443. https://doi.org/10.1007/s00397-015-0842-2

Kawabata A, Akuzawa S, Ishii Y, Yazaki T, and Otsubo Y (1996) Sol-gel transition and elasticity of starch. Biosci Biotech Biochem 60(4):567-570. https://doi.org/10.1271/bbb.60.567

Kim OV, Litvinov RI, Weisel JW, Alber MS (2014) Structural basis for the non linear mechanics of fibrin networks under compression. Biomaterials 35(25):6739-6749. https://doi.org/10.1016/j.biomaterials.2014.04.056

Kirkpatrick FH, Dumais MM, White HW, Guiseley KB (1993) Influence of the agarose matrix in pulsed-field electrophoresis. Electrophoresis 14(1):349-354. https://doi.org/10.1002/elps.1150140159

Kunitz M (1928) Syneresis and swelling of gelatin. The Journal of General Physiology 12(2):289-312. https://doi.org/10.1085/jgp.12.2.289

Lang NR, Münster S, Metzner C, Krauss P, Schürmann S, Lange J, Aifantis KE, Friedrich O, and Fabry B (2013) Estimating the 3D pore size distribution of biopolymer networks from directionally biased date. Biophys J 105(9):1967-1975. https://doi.org/10.1016/j.bpj.2013.09.038

Laurent TC (1967) Determination of the structure of agarose gels by gel permeation chromatography. Biochim Biophys Acta 136(2):199-205. https://doi.org/10.1016/0304-4165(67)90064-5

Maaloum M, Pernodet N Tinland B (1998) Agarose gel structure using atomic force microscopy: Gel concentration and ionic strength effects. Electrophoresis 19(10):1606-1610. https://doi.org/10.1002/elps.1150191015

Manno M, and Palma MU (1997) Fractal morphogenesis and interacting processes in gelation. PRL 79(21):4286-4288. https://doi.org/10.1103/PhysRevLett.79.4286

Mao B, Divoux T, and Snabre P (2016) Normal force controlled rheology applied to agar gelation. J Rheol 60(3):473-489. https://doi.org/10.1122/1.4944994

Mao, B, Divoux T, and Snabre P (2017a) Impact of saccharides on the drying kinetics of agarose gels measured by in-situ interferometry. Sci Rep 7:41185. https://doi.org/10.1038/srep41185 
Mao B, Bentaleb A, Louerat F, Divoux T, and Snabre P (2017b) Heat-induced aging of agar solutions: Impact on the structural and mechanical properties of agar gels. Food Hydrocolloids 64:59-69. https://doi.org/10.1016/j.foodhyd.2016.10.020

Mao B, Bouchaudy A, Salmon JB, Divoux T, and Snabre P (2017c) Time-resoved rheological monitoring of viscoelastic materials under drying. AERC 2017. 11th Annual European Rheology Conference, Copenhagen, Denmark. Abstract number GS19. https://nordicrheologysociety.org/content/aerc/2017/images/AERC2017AbstractBook-20170322.pdf. Accessed 24 november 2020.

Matsuhashi T (1990). Agar. In: Harris P (ed) Food gels. Elsevier Applied Science, New York, pp 1-51. ISBN 978-94-009-0755-3

Meunier V, Nicolai T, and Durand D (2000) Structure and kinetics of aggregating $\kappa$-carrageenan studied by light scattering. Macromolecules 33(7):2497-2504. https://doi.org/10.1021/ma991433t

Mezzenga R, Schurtenberger P, Burbidge A, and Michel M (2005) Understanding foods as soft materials. Nat Mat 4:729-740. https://doi.org/10.1038/nmat1496

Mitsuiki M, Mizuno A, and Motoki M (1999) Determination of molecular weight of agars and effect of the molecular weight on the glass transition. J Agric Food Chem 47(2):473-478. https://doi.org/10.1021/jf980713p

Mohammed ZH, Hember MWN, Richardson RK, Morris ER (1998) Kinetic and equilibrium processes in the formation and melting of agarose gels. Carbohydrate Polymers 36(1):15-26 https://doi.org/10.1016/S01448617(98)00011-3

Molginer A, Rubinstein B (2005) The physics of filipodial protrusion. Biophys J 89(2):782-795 https://doi.org/10.1529/biophysj.104.056515

Morita T, Narita T, Mukai S, Yanagisawa M, and Tokita M (2013) Phase behaviors of agarose gel. AIP Advances 3(4):042128:1-12. https://doi.org/10.1063/1.4802968

Nakamura K, Shinoda E, Tokita M (2001) The influence of compression velocity on strength and structure for gellan gels. Food Hydrocolloids 15(3):247-252. https://doi.org/10.1016/S0268-005X(01)00021-2

Nitta T, Endo Y, Haga H, and Kawabata K (2003) Microdomain structure of agar gels observed by mechanicalscanning probe microscopy. J Electron Microsc 52(3):277-281. https://doi.org/10.1093/jmicro/52.3.277

Normand V, Lootens DL, Amici E, Plucknett KP, and Aymard P (2000) New insight into agarose gel mechanical properties. Biomacromolecules 1(4):730-738. https://doi.org/10.1021/bm005583j

Normand V, Aymard P, Lootens DL, Amici E, Plucknett KP, Frith WJ (2003) Effect of sucrose on agarose gels mechanical behaviour. Carbohydrate Polymers 54(1):83-95. https://doi.org/10.1016/S0144-8617(03)00153-X

Ogston AG (1958) The spaces in a uniform random suspension of fibres. Trans Faraday Soc 54:1754-1757. https://doi.org/10.1039/TF9585401754

Ouellet S, Cronin D, Worswick M (2006) Compressive response of polymeric foams under quasi-static, medium and high strain rate conditions. Polymer Testing 25(6):731-743. https://doi.org/10.1016/j.polymertesting.2006.05.005

Pernodet N, Maaloum M, Tinland B (1997) Pore size of agarose gels by atomic force microscopy. Electrophoresis 18(1):55-58. https://doi.org/10.1002/elps.1150180111

Ramzi M, Rochas C, and Guenet JM (1998) Structure-properties relation for agarose thermoreversible gels in binary solvents. Macromolecules 31(18):6106-6111. https://doi.org/10.1021/ma9801220

Righetti G, Brost BCW, and Snyder RS (1981) On the limiting pore size of hydrophilic gels for electrophoresis and isoelectric focussing. J BiochemBiophys Methods 4(5-6):347-363. https://doi.org/10.1016/0165$022 \times(81) 90075-0$

Rinaudo M (2008) Main properties and current applications of some polysaccharides as biomaterials. Polym Int 57(3):397-430. https://doi.org/10.1002/pi.2378 
Rochas C, Brûlet A, and Guenet JM (1994) Thermally reversible gelation of agarose in water/dimethyl sulfoxide mixtures. Macromolecules 27(14):3830-3835. https://doi.org/10.1021/ma00092a023

Rochas C, Hecht AM, Geissler E (1996) Swelling properties of agarose gels. J Chim Phys 93:850-857. https://doi.org/10.1051/jcp/1996930850

Sahimi M (1986) Relation between the critical exponent of elastic percolation networks and the conductivity and geometrical exponents. J Phys C: Solid State Phys 19(4):L79-L83. https://doi.org/10.1088/0022-3719/19/4/004

San Biagio PL, Madonia F, Sciortino F, Palma-Vitorelli MB, et Palma MU (1984) Cooperative interaction of polysaccharide molecules in water: A role of connectivity properties of H-Bonds within the solvent? J Phys 45(C7):225-233. https://doi.org/10.1051/jphyscol:1984725

San biagio PL, Bulone D, Emanuele A, Palma-Vitorelli MB, Palma MU (1996) Spontaneous symmetry-breaking pathways: time-resolved study of agarose gelation. Food Hydrocolloids 10(1):91-97. https://doi.org/10.1016/S0268-005X(96)80059-2

Schafer SE, Stevens ES (1995) A reexamination of the double-helix model for agarose gels using optical rotation. Biopolymers 36(1):103-108. https://doi.org/10.1002/bip.360360109

Schneider CA, Rasband WS, and Eliceiri KW (2012) NIH Image to ImageJ: 25 years of image analysis. Nat Methods 9:671-675. https://doi.org/10.1038/nmeth.2089

Stanley NF (2006) Agars. In: Stephen AM, Philips GO (ed) Food polysaccharides and their Applications, 2nd edn. Boca Raton, CRC Press, pp 217-238. ISBN 9780429116162

Strange DGT, Fletcher TL, Tonsomboon K, Brawn H, Zhao X, and Oyen ML (2013) Separating poroviscoelastic deformation mechanisms in hydrogels. Appl Phys Lett 102:03913. https://doi.org/10.1063/1.4789368

Tokita M, and Hikichi K (1987) Mechanical studies of sol-gel transition: Universal behaviour of elastic modulus. Phys Rev A 35(10):4329-4333. https://doi.org/10.1103/physreva.35.4329

van Oosten ASG, Vahabi M, Licup AJ, Sharma A, Galie PA, MacKintosh FC, and Janmey PA (2016) Uncoupling shear and uniaxial elastic moduli of semiflexible biopolymer networks: compression-softening and stretch-stiffening. Sci rep $6: 19270$. https://doi.org/10.1038/srep19270

von Terzaghi K (1925) Erdbaumechanik auf bodenphysikalischer Grundlage. Leipzig and Vienna. Franz Deuticke. 399 pages.

Waki S, Harvey JD, Bellamy AR (1982) Study of agarose gels by electron microscopy of freeze-fractured surfaces. Biopolymers 21(9):1909-1926. https://doi.org/10.1002/bip.360210917

Watase M, and Nishinari K (1983) Rheological properties of agarose gels with different molecular weights. Rheol Acta 22(6):580-587. https://doi.org/10.1007/BF01351404

Wen Q, Basu A, Janmey PA, and Yodh AG (2012) Non-affine deformations in polymer hydrogels. Soft Matter 8(31):8039-8049. https://doi.org/10.1039/c2sm25364j

Xiong JY, Narayanan J, Liu XY, Chong TK, Chen SB, and Chung TS (2005) Topology evolution and gelation mechanism of agarose gel. J Phys Chem B 109(12):5638-5643. https://doi.org/10.1021/jp044473u

Yamaue T and Doi M (2004) Swelling dynamics of constrained thin-plate gels under an external force. Phys Rev E Stat Nonlin Soft Matter Phys 70(1 Pt 1):011401. https://doi.org/10.1103/PhysRevE.70.011401

Yamaue T and Doi M (2005) The stress diffusion coupling in the swelling dynamics of cylindrical gels. J Chem Phys 122:084703. https://doi.org/10.1063/1.1849153

Zhang Y, Fu X, Duan D, Xu J, and Gao X (2019) Preparation and characterization of agar, agarose, and agaropectin from the red alga Ahnfeltia plicata. J Ocean Limnol 37(3):815-824. https://doi.org/10.1007/s00343019-8129-6 